Original Research Paper

\title{
SIR Transmission Model of Dengue Virus Taking Into Account Two Species of Mosquitoes and an Age Structure in the Human Population
}

\author{
${ }^{1}$ R. Sungchasit, ${ }^{1}$ P. Pongsumpun and ${ }^{2,3}$ I.M. Tang \\ ${ }^{I}$ Department of Mathematics, Faculty of Science, King Mongkut's Institute of Technology Ladkrabang, \\ Chalongkrung Road, Ladkrabang, Bangkok, 10520, Thailand \\ ${ }^{2}$ Department of Materials Science, Faculty of Science, Kasetsart University, 50 Ngam Wong Wan Road, \\ Lat Yao Chatuchak, Bangkok 10900, Thailand \\ ${ }^{3}$ Department of Physics, Faculty of Science, Mahidol University, Rama 6 road, Bangkok 10400, Thailand
}

\author{
Article history \\ Received: 30-07-2014 \\ Revised: $10-05-2015$ \\ Accepted: 22-06-2015 \\ Corresponding Author: \\ P. Pongsumpun \\ Department of Mathematics, \\ Faculty of Science, King \\ Mongkut's Institute of \\ Technology Ladkrabang, \\ Chalongkrung Road, \\ Ladkrabang, Bangkok, 10520, \\ Thailand \\ Email: kppuntan@kmitl.ac.th
}

\begin{abstract}
Dengue is a vector-borne disease. It is transmitted to humans by the bites of the Aedes aegypti and Aedes albopictus mosquitoes. The human population is separated into two classes, a child class and an adult class, each class being described by a SIR model. The transmission rates of the two mosquito species are different and depend on what class the humans belong to. We develop a single model taking into account the presence of two type of mosquitoes and two age classes and apply it to dengue fever. The model shows how it is possible for the maximum level of infected human to be reached in a short time. The nature of stability of the equilibrium state and the trajectories of the individual classes in the model are determined by the values of the basic reproduction number by setting the values of the parameters in the model to different values which reflect the environment in which the epidemic is occurring in the model.
\end{abstract}

Keywords: Aedes Aegypti, Aedes Albopictus, Dengue Disease, Endemic Disease State, Equilibrium State, SIR Model

\section{Introduction}

Dengue fever is regarded as a serious infectious disease that risks about 2.5 billion people around the world, especially in tropical countries. It is a major epidemic disease occurred in Southeast Asia. Such epidemic arises due to climate change, knowledge of people and awareness on dengue fever so as to the dengue fever possibly become an endemic for a long time. Moreover, World Health Organization (WHO) (WHO, 2009) estimated 50 to 100 million cases worldwide. About 500,000 people are estimated to be infected by dengue hemorrhagic fever each year.

Dengue fever is caused by four serotypes and they are closely related as a family of dengue virus 1 (DEN 1), virus 2 (DEN2), virus 3 (DEN3) and virus 4 (DEN4). There are viruses carried by two kinds of mosquitoes such as Aedes aegypti and Aedes albopictus. This disease is transmitted to the human though biting of mosquitoes. Recently, It was detected in Asia. However, Aedes aegypti is still the principal vector of dengue fever transmission. Another interesting fact is the shift of patients' phenomena where dengue fever previously attacks children of primary school age, but now everybody is vulnerable to fever (Pongsumpun and Tang, 2001; Syafruddin and Noorani, 2012).

Dengue virus is transmitted between the human by biting of an infected Aedes mosquito. When a vector bites someone who be infected with dengue virus, the virus is transferred to mosquitoes and it become infected mosquito. After the infected vector bites the susceptible human, then the virus move into the human bloodstream and it spreads throughout the body. Usually, the mosquitoes bite susceptible people during the day time. Dengue fever is the most common disease in urban areas. The outbreaks commonly occur during the rainy season when the mosquitoes heavily breed in standing water. The dengue fever cases are increasing worldwide. The complications of the disease are leading cases of serious illness and most death in children (Kerpaninch et al., 2001; Kabilan et al., 2003; Malhotra et al., 2006; Wiwanitkit, 2006; Pongsumpum, 2011; Joshi et al., 2002; Koenraadt et al., 2007). One of the major public health problems in many tropical and subtropical regions where Aedes aegypti and Aedes albopictus are present. 
It is noted that Aedes albopictus was the principal vector in the $1940 \mathrm{~s}$ outbreaks in Japan (Hotta, 1998), whereas, Aedes aegypti is commonly the principal dengue vector in the tropical and subtropical regions. Aedes aegypti is highly domesticated and exhibits strong anthropophilia.

Traditional modeling in epidemiology focus on stability equilibria, since this characterizes if a disease will become endemic and this is a major concern for public health officers. The concept of a basic reproductive number $\left(R_{0}\right)$ was introduced and became a modeling paradigm (Smith et al., 2012) for a very recent review on the works by Ross and Macdonald from a medical modeling point of view. In a fairly large class of models, we can define $\mathrm{R}_{0}$ unambiguously and it can be shown that if $\mathrm{R}_{0}<1$, the disease is extinct while if $\mathrm{R}_{0}>1$ it becomes endemic (Diekmann and Heesterbeek, 2000).

Hence, in this study, we analyze the SIR (Susceptible-Infected-Recovered) equations for human and SI (Susceptible-Infected) equations for mosquitoes. The model will apply empirically on data of dengue patients reports by Ministry of Public Health, Thailand (2002-2012) as shown in Fig. 1. The purpose of this paper is to study the age structural model of dengue disease incorporated the influence of Aedes aegypti and Aedes albopictus.

\section{Mathematical Model}

The SIR and SI simulates the spread of dengue virus between host and vector populations. The model is based on the Susceptible, Infected and Recovered (SIR) model of infected disease epidemiology, which was adopted by (Nuraini et al., 2007; Yaacob, 2007). The age structure is introduced into a model, i.e., children and adults, then we modify it by incorporating the different behaviors of Aedes aegypti and Aedes albopictus. In Fig. 1, we show the age distribution of the incidence rates in one province in Thailand during 2002-2012 epidemic. As we see, most cases occur in children under the age of 15. However, a small number of cases do occur in older people. Similar distributions are seen in the other provinces in the country.

This model with age structure, the dynamics of each component of the human is given by:

$$
\begin{aligned}
& \frac{d S_{c}}{d t}=P_{c} N_{t c}-\beta_{a c}\left(1+\alpha_{a} \sin \varepsilon t\right) \\
& I_{v a 1} S_{c}-\beta_{b c}\left(1+\alpha_{b} \sin \varepsilon t\right) I_{v b 1} S_{c}-\mu_{d} S_{c} \\
& \frac{d I_{c 1}}{d t}=\beta_{a c}\left(1+\alpha_{a} \sin \varepsilon t\right) I_{v a 1} S_{c}-\kappa_{c 1} I_{c 1}-\mu_{d} I_{c 1} \\
& \frac{d I_{c 2}}{d t}=\beta_{b c}\left(1+\alpha_{b} \sin \varepsilon t\right) I_{v b 1} S_{c}-\kappa_{c 2} I_{c 2}-\mu_{d} I_{c 2} \\
& \frac{d R_{c}}{d t}=\kappa_{c 1} I_{c 1}+\kappa_{c 2} I_{c 2}-\mu_{d} R_{c} \\
& \frac{d S_{a}}{d t}=P_{a} N_{t a}-\beta_{a a}\left(1+\alpha_{a} \sin \varepsilon t\right) \\
& I_{v a 2} S_{a}-\beta_{b a}\left(1+\alpha_{b} \sin \varepsilon t\right) I_{v b 2} S_{a}-\mu_{d} S_{a} \\
& \frac{d I_{a 1}}{d t}=\beta_{a a}\left(1+\alpha_{a} \sin \varepsilon t\right) I_{v a 2} S_{a}-\kappa_{a 1} I_{a 1}-\mu_{d} I_{a 1} \\
& \frac{d I_{a 2}}{d t}=\beta_{b a}\left(1+\alpha_{b} \sin \varepsilon t\right) I_{v b 2} S_{a}-\kappa_{a 2} I_{a 2}-\mu_{d} I_{a 2} \\
& \frac{d R_{a}}{d t}=\kappa_{a 1} I_{a 1}+\kappa_{a 2} I_{a 2}-\mu_{d} R_{a}
\end{aligned}
$$

where the variables and parameters are defined in Table 1.

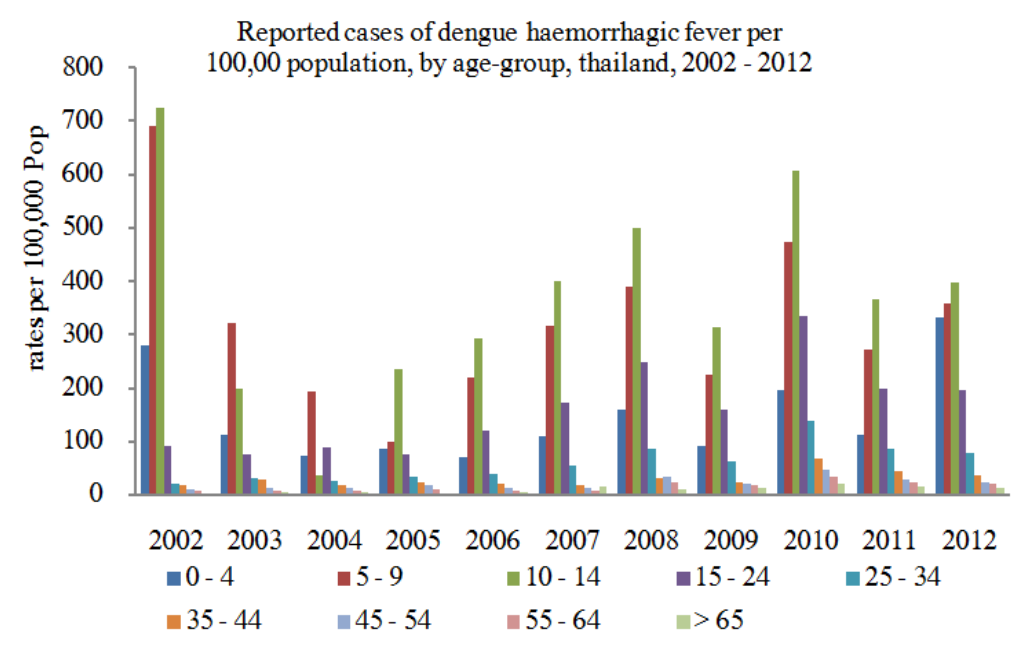

Fig. 1. Age distribution of the 2002-2012 Dengue fever incidence rates in Thailand 
Table 1. Parameters for equations (1a)-(1h) and their definitions

\begin{tabular}{l}
$\frac{\text { Variable/para }}{\mathrm{S}_{\mathrm{c}}, \mathrm{I}_{\mathrm{c} 1}, \mathrm{I}_{\mathrm{c} 2}, \mathrm{R}_{\mathrm{c}}}$ \\
$\mathrm{S}_{\mathrm{a}}, \mathrm{I}_{\mathrm{a} 1}, \mathrm{I}_{\mathrm{a} 2}, \mathrm{R}_{\mathrm{a}}$ \\
$\mathrm{N}_{\mathrm{t}}$ \\
$\mathrm{N}_{\mathrm{tc}}$ and $\mathrm{N}_{\mathrm{ta}}$ \\
$\mathrm{P}_{\mathrm{c}}$ and $\mathrm{P}_{\mathrm{a}}$ \\
$\beta_{\mathrm{ac}}$ \\
$\beta_{\mathrm{bc}}$ \\
$\beta_{\mathrm{aa}}$ \\
$\beta_{\mathrm{ba}}$ \\
$\kappa_{\mathrm{c} 1}$ \\
$\kappa_{\mathrm{c} 2}$ \\
$\kappa_{\mathrm{a} 1}$ \\
$\kappa_{\mathrm{a} 2}$ \\
$\mu_{\mathrm{d}}$ \\
$\mathrm{a}_{\mathrm{a}}$ \\
$\mathrm{a}_{\mathrm{b}}$ \\
$\rho_{\mathrm{va}}$ \\
$\rho_{\mathrm{vb}}$ \\
\hline
\end{tabular}

\section{Definition}

The numbers of susceptible children, infected from Aedes

aegypti and Aedes albopictus in children and recovered children

The numbers of susceptible adult, infected from Aedes aegypti and

Aedes albopictus in adult and recovered adult

The total population

The total population in children and the total population in adult they are constant variables

The birth rate of children and adult human

The transmission probability of dengue virus from Aedes aegypti to children

The transmission probability of dengue virus from Aedes albopictus to children

The transmission probability of dengue virus from Aedes aegypti to adult

The transmission probability of dengue virus from Aedes albopictus to adult

The rate at which the infected children from Aedes aegypti can recover

The rate at which the infected children from Aedes albopictus can recover

The rate at which the infected adult from Aedes aegypti can recover

The rate at which the infected adult from Aedes albopictus can recover

The natural death rate of human

The measure of influence on the transmission process from Aedes aegypti mosquito to human

The measure of influence on the transmission process from Aedes albopictus to human

The measure of influence on the transmission process from Aedes aegypti to human The measure of influence on the transmission process from Aedes albopictus to human
It we add Equation (1a) - (1h) together, we get:

$$
\begin{aligned}
& \frac{d N_{t}}{d t}=\frac{d N_{t c}}{d t}+\frac{d N_{t a}}{d t} \\
& =\left(S_{c}+I_{c 1}+I_{c 2}+R_{c}\right)+\left(S_{a}+I_{a 1}+I_{a 2}+R_{a}\right)
\end{aligned}
$$

The total children and adult populations are supposed to have constant sizes, i.e., $\frac{d N_{t c}}{d t}=0$ and $\frac{d N_{t a}}{d t}=0$, the birth rate would have to be equivalent to the death rate, $P_{c}=P_{a}=\mu_{d}$ in children and adult, respectively.

where, $N_{t c}$ is the total number of children and is equivalent to $S_{c}+I_{c 1}+I_{c 2}+R_{c}, N_{t a}$, the total population in adult and is equal to $S_{a}+I_{a 1}+I_{a 2}+R_{a}$.

The dynamics of mosquitoes is described by:

$$
\begin{aligned}
& \frac{d S_{v a 1}}{d t}=A_{v_{a}}-\lambda_{v a 1}\left(1+\rho_{v a} \sin \varepsilon t\right) I_{c 1} S_{v a 1}-\mu_{v_{a}} S_{v a 1} \\
& \frac{d I_{v a 1}}{d t}=\lambda_{v a 1}\left(1+\rho_{v a} \sin \varepsilon t\right) I_{c 1} S_{v a 1}-\mu_{v_{a}} I_{v a 1} \\
& \frac{d S_{v a 2}}{d t}=A_{v_{a}}-\lambda_{v a 2}\left(1+\rho_{v a} \sin \varepsilon t\right) I_{a 1} S_{v a 2}-\mu_{v_{a}} S_{v a 2} \\
& \frac{d I_{v a 2}}{d t}=\lambda_{v a 2}\left(1+\rho_{v a} \sin \varepsilon t\right) I_{a 1} S_{v a 2}-\mu_{v_{a}} I_{v a 2} \\
& \frac{d S_{v b 1}}{d t}=A_{v_{b}}-\lambda_{v b 1}\left(1+\rho_{v b} \sin \varepsilon t\right) I_{c 2} S_{v b 1}-\mu_{v_{b}} S_{v b 1}
\end{aligned}
$$

$$
\begin{aligned}
& \frac{d I_{v b 1}}{d t}=\lambda_{v b 1}\left(1+\rho_{v b} \sin \varepsilon t\right) I_{c 2} S_{v b 1}-\mu_{v_{b}} I_{v b 1} \\
& \frac{d S_{v b 2}}{d t}=A_{v_{b}}-\lambda_{v b 2}\left(1+\rho_{v b} \sin \varepsilon t\right) I_{a 2} S_{v b 2}-\mu_{v_{b}} S_{v b 2}
\end{aligned}
$$

$\frac{d I_{v b 2}}{d t}=\lambda_{v b 2}\left(1+\rho_{v b} \sin \varepsilon t\right) I_{a 2} S_{v b 2}-\mu_{v_{b}} I_{v b 2}$

where, the variable and parameters are given in Table 2. If we add Equation (2a)-(2h) together, we get:

$\frac{d\left(S_{v a 1}+I_{v a 1}\right)}{d t}=A_{v_{a}}-\mu_{v_{a}} N_{v a 1}$

$\frac{d\left(S_{v a 2}+I_{v a 2}\right)}{d t}=A_{v_{a}}-\mu_{v_{a}} N_{v a 2}$

$\frac{d\left(S_{v b 1}+I_{v b 1}\right)}{d t}=A_{v_{b}}-\mu_{v_{b}} N_{v b 1}$

$\frac{d\left(S_{v b 2}+I_{v b 2}\right)}{d t}=A_{v_{b}}-\mu_{v_{b}} N_{v b 2}$

where, $N_{v a 1}$ and $N_{v a 2}$ are the numbers of Aedes aegypti in children and adult respectively, which is equal to $\mathrm{S}_{\mathrm{va} 1}+\mathrm{I}_{\mathrm{va} 1}$ and $\mathrm{S}_{\mathrm{va} 2}+\mathrm{I}_{\mathrm{va} 2} . \mathrm{N}_{\mathrm{vb} 1}$ and $\mathrm{N}_{\mathrm{vb} 2}$ are the numbers of Aedes albopictus in children and adult respectively, which is equal to $S_{v b 1}+I_{v b 1}$ and $S_{v b 2}+I_{v b 2}$. If the numbers of mosquitoes are also constant each other (3a)(3d) gives $N_{v a 1}=A_{v_{a}} / \mu_{v_{a}}, N_{v a 2}=A_{v_{a}} / \mu_{v_{a}}, N_{v b 1}=A_{v_{b}} / \mu_{v_{b}}$ and $N_{v b 2}=A_{v_{b}} / \mu_{v_{b}}$. 
Table 2. Parameters for equations (2a)-(2g) and their definitions

\begin{tabular}{ll}
\hline Variable/ parameter & Definition \\
\hline $\mathrm{S}_{\mathrm{va} 1}$ and $\mathrm{I}_{\mathrm{va} 1}$ & The number of susceptible and infected Aedes aegypti mosquitoes who be infected from children \\
$\mu_{v_{a}}$ & The death rate of Aedes aegypti mosquito \\
$A_{v_{a}}$ & The carrying capacity of the environment for Aedes aegypit \\
$\lambda_{\mathrm{va} 1}$ & The probability that a dengue virus transmitted to the Aedes aegypti from an infected children \\
$\mathrm{S}_{\mathrm{va2}}$ and $\mathrm{I}_{\mathrm{va} 2}$ & The number of susceptible and infected Aedes aegypti mosquitoes who be infected from adult \\
$\mu_{v_{a}}$ & The death rate of Aedes aegypti mosquito \\
$A_{v_{a}}$ & The carrying capacity of the environment for Aedes aegypit mosquito \\
$\lambda_{\mathrm{va} 2}$ & The probability that a dengue virus transmitted to the Aedes aegypti from an infected adult human \\
$\mathrm{S}_{\mathrm{vb} 1}$ and $\mathrm{I}_{\mathrm{vb} 1}$ & The number of susceptible and infected Aedes albopictus mosquitoes who be infected from children \\
$\mu_{v_{b}}$ & The death rate of Aedes albopictus mosquito \\
$A_{v_{b}}$ & The carrying capacity of the environment for Aedes albopictus mosquito \\
$\lambda_{\mathrm{vb} 1}$ & The probability that a dengue virus transmitted to the Aedes albopictus from an infected human in children \\
$\mathrm{S}_{\mathrm{vb} 2}$ and $\mathrm{I}_{\mathrm{vb} 2}$ & The number of susceptible and infected Aedes albopictus mosquitoes who be infected from adult human \\
$\mu_{v_{b}}$ & The death rate of Aedes albopictus mosquito \\
$A_{v_{b}}$ & The carrying capacity of the environment for Aedes albopictus mosquito \\
$\lambda_{\mathrm{vb} 2}$ & The probability that a dengue virus transmitted to the Aedes albopictus from an infected adult
\end{tabular}

We normalize parameter (1a)-(1h) and (2a)-(2h) by writing $S_{c}^{\prime}=\frac{S_{c}}{N_{t c}}, I_{c 1}^{\prime}=\frac{I_{c 1}}{N_{t c}}, I_{c 2}^{\prime}=\frac{I_{c 2}}{N_{t c}}, R_{c}^{\prime}=\frac{R_{c}}{N_{t c}}$ in children and $\quad S_{a}^{\prime}=\frac{S_{a}}{N_{t a}}, \quad I_{a 1}^{\prime}=\frac{I_{a 1}}{N_{t a}}, \quad I_{a 2}^{\prime}=\frac{I_{a 2}}{N_{t a}}, \quad R_{a}^{\prime}=\frac{R_{a}}{N_{t a}} \quad$ in adult. $S_{v a 1}^{\prime}=\frac{S_{v a 1}}{N_{v a 1}}, I_{v a 1}^{\prime}=\frac{I_{v a 1}}{N_{v a 1}}, S_{v a 2}^{\prime}=\frac{S_{v a 2}}{N_{v a 2}}, I_{v a 2}^{\prime}=\frac{I_{v a 2}}{N_{v a 2}}$, $S_{v b 1}^{\prime}=\frac{S_{v b 1}}{N_{v b 1}}, I_{v b 1}^{\prime}=\frac{I_{v b 1}}{N_{v b 1}}, S_{v b 2}^{\prime}=\frac{S_{v b 2}}{N_{v b 2}}$ and $I_{v b 2}^{\prime}=\frac{I_{v b 2}}{N_{v b 2}}$, then the reduced equations become:

$$
\begin{aligned}
& \frac{d}{d t} S_{c}^{\prime}=\mu_{d}-\beta_{a c}\left(1+\alpha_{a} \sin \varepsilon t\right) \\
& I_{v a 1}^{\prime} N_{v a 1} S_{c}^{\prime}-\beta_{b c}\left(1+\alpha_{b} \sin \varepsilon t\right) I_{v b 1}^{\prime} N_{v b 1} S_{c}^{\prime}-\mu_{d} S_{c}^{\prime} \\
& \frac{d}{d t} I_{c 1}^{\prime}=\beta_{a c}\left(1+\alpha_{a} \sin \varepsilon t\right) I_{v a 1}^{\prime} N_{v a 1} S_{c}^{\prime}-\kappa_{c 1} I_{c 1}^{\prime}-\mu_{d} I_{c 1}^{\prime} \\
& \frac{d}{d t} I_{c 2}^{\prime}=\beta_{b c}\left(1+\alpha_{b} \sin \varepsilon t\right) I_{v b 1}^{\prime} N_{v b 1} S_{c}^{\prime}-\kappa_{c 2} I_{c 2}^{\prime}-\mu_{d} I_{c 2}^{\prime} \\
& \frac{d}{d t} S_{a}^{\prime}=\mu_{d}-\beta_{a a}\left(1+\alpha_{a} \sin \varepsilon t\right) \\
& I_{v a 2}^{\prime} N_{v a 2} S_{a}^{\prime}-\beta_{b a}\left(1+\alpha_{b} \sin \varepsilon t\right) I_{v b 2}^{\prime} N_{v b 2} S_{a}^{\prime}-\mu_{d} S_{a}^{\prime} \\
& \frac{d}{d t} I_{a 1}^{\prime}=\beta_{a a}\left(1+\alpha_{a} \sin \varepsilon t\right) I_{v a 2}^{\prime} N_{v a 2} S_{a}^{\prime}-\kappa_{a 1} I_{a 1}^{\prime}-\mu_{d} I_{a 1}^{\prime} \\
& \frac{d}{d t} I_{a 2}^{\prime}=\beta_{b a}\left(1+\alpha_{b} \sin \varepsilon t\right) I_{v b 2}^{\prime} N_{v b 2} S_{a}^{\prime}-\kappa_{a 2} I_{a 2}^{\prime}-\mu_{d} I_{a 2}^{\prime} \\
& \frac{d}{d t} I_{v a 1}^{\prime}=\lambda_{v a 1}\left(1+\rho_{v a} \sin \varepsilon t\right) I_{c 1}^{\prime} N_{t c} S_{v a 1}^{\prime}-\mu_{v a} I_{v a 1}^{\prime}
\end{aligned}
$$

$$
\frac{d}{d t} I_{v a 2}^{\prime}=\lambda_{v a 2}\left(1+\rho_{v a} \sin \varepsilon t\right) I_{a 1}^{\prime} N_{t a} S_{v a 2}^{\prime}-\mu_{v_{a}} I_{v a 2}^{\prime}
$$

$\frac{d}{d t} I_{v b 1}^{\prime}=\lambda_{v b 1}\left(1+\rho_{v b} \sin \varepsilon t\right) I_{c 2}^{\prime} N_{t c} S_{v b 1}^{\prime}-\mu_{v_{b}} I_{v b 1}^{\prime}$

$$
\frac{d}{d t} I_{v b 2}^{\prime}=\lambda_{v b 2}\left(1+\rho_{v b} \sin \varepsilon t\right) I_{a 2}^{\prime} N_{t a} S_{v b 2}^{\prime}-\mu_{v_{b}} I_{v b 2}^{\prime}
$$

Where:

$$
\begin{aligned}
& S_{c}^{\prime}+I_{c 1}^{\prime}+I_{c 2}^{\prime}+R_{c}^{\prime}=1, S_{a}^{\prime}+I_{a 1}^{\prime}+I_{a 2}^{\prime}+R_{a}^{\prime}=1, \\
& S_{v a 1}+I_{v a 1}=1, S_{v a 2}^{\prime}+I_{v a 2}^{\prime}=1, S_{v b 1}^{\prime}+I_{v b 1}^{\prime}=1 \text { and } S_{v b 2}^{\prime}+I_{v b 2}^{\prime}=1
\end{aligned}
$$

\section{Mathematical Analysis}

\section{Equilibrium States}

The equilibrium states $\left(S_{c}^{*}, I_{c 1}^{*}, I_{c 2}^{*}, S_{a}^{*}, I_{a 1}^{*}, I_{a 2}^{*}, I_{v a 1}^{*}, I_{v b 1}^{*}, I_{v a 2}^{*}, I_{v b 2}^{*}\right)$ are obtained by setting the right hand side of $(4 a)-(4 j)$ to zero. Therefore we obtain $\left(S_{c}^{*}, I_{c 1}^{*}, I_{c 2}^{*}, I_{v a 1}^{*}, I_{v b 1}^{*}\right)$ and $\left(S_{a}^{*}, I_{a 1}^{*}, I_{a 2}^{*}, I_{v a 2}^{*}, I_{v b 2}^{*}\right)$. Doing this, we get four equilibrium states.

A. The two group disease free equilibrium state:

$$
E_{0}=(1,0,0,1,0,0,0,0,0,0)
$$

A1.the disease free equilibrium state:

$$
E_{o c}=\left(S_{c}^{*}, I_{c l}^{*}, I_{c 2}^{*}, I_{v a l}^{*}, I_{v b 1}^{*}\right)=(1,0,0,0,0) \text { in children }
$$

A2.the disease free equilibrium state: 


$$
E_{0 a}=\left(S_{a}^{*}, I_{a 1}^{*}, I_{a 2}^{*}, I_{v a 2}^{*}, I_{v b 2}^{*}\right)=(1,0,0,0,0) \text { in adult }
$$

B. The two group endemic equilibrium state:

$$
\hat{E}=\left(S_{c}^{*}, I_{c 1}^{*}, I_{c 2}^{*}, I_{v a 1}^{*}, I_{v b 1}^{*}, S_{a}^{*}, I_{a 1}^{*}, I_{a 2}^{*}, I_{v a 2}^{*}, I_{v b 2}^{*}\right)
$$

B1.the endemic state:

$$
E_{l c}=\left(S_{c}^{*}, I_{c l}^{*}, I_{c 2}^{*}, I_{v a l}^{*}, I_{v b l}^{*}\right) \text { in children }
$$

Where:

$$
\begin{aligned}
& \left(N_{t c} \mu_{d} \lambda_{v b 1}+\left(\kappa_{c 2}+\mu_{d}\right) \mu_{v b 1}\right. \\
& S_{c}^{*}=\frac{\left.+N_{t c} \mu_{d} \lambda_{v b 1} \rho_{v b} \sin \varepsilon t\right)}{\left(N _ { t c } \lambda _ { v b 1 } \left(I_{v a 1}^{*} N_{v a 1} \beta_{a c}+N_{v b 1} \beta_{b c}+\mu_{d}+\left(I_{v a 1}^{*} N_{v a 1}\right.\right.\right.} \\
& \left.\left.\left.\left.\alpha_{a} \beta_{a c}+N_{v b 1} \alpha_{b} \beta_{b c}\right) \sin \varepsilon t\right)\left(1+\rho_{v b} \sin \varepsilon t\right)\right)\right) \\
& I_{v a 1}^{*} N_{v a 1} \beta_{a c}\left(1+\alpha_{a} \sin \varepsilon t\right)\left(N_{t c} \mu_{d} \lambda_{v b 1}\right. \\
& I_{c 1}^{*}=\frac{\left.\left.+\left(\kappa_{c 2}+\mu_{d}\right) \mu_{v b}+N_{t c} \mu_{d} \lambda_{v b 1} \rho_{v b} \sin \varepsilon t\right)\right)}{\left(N _ { t c } \lambda _ { b b 1 } ( \kappa _ { c 1 } + \mu _ { d } ) \left(I_{v a 1}^{*} N_{v a 1} \beta_{a c}+N_{v b 1} \beta_{b c}+\mu_{d}+\right.\right.} \\
& \left.\left.\left(I_{v a 1}^{*} N_{v a 1} \alpha_{a} \beta_{a c}+N_{v b 1} \alpha_{b} \beta_{b c}\right) \sin \varepsilon t\right)\left(1+\rho_{v b} \sin \varepsilon t\right)\right) \\
& \left(I _ { v b 1 } ^ { * } N _ { v b 1 } \beta _ { b c } ( 1 + \alpha _ { b } \operatorname { s i n } \varepsilon t ) \left(N_{t c} \lambda_{v b 1} \mu_{d}\right.\right. \\
& I_{c 2}^{*}=\frac{\left.\left.+\left(\kappa_{c 2}+\mu_{d}\right) \mu_{v b}+N_{t c} \lambda_{v b 1} \mu_{d} \rho_{v b} \sin \varepsilon t\right)\right)}{\left(N _ { t c } \lambda _ { v b 1 } ( \kappa _ { c 2 } + \mu _ { d } ) \left(I_{v a 1}^{*} N_{v a 1} \beta_{a c}+I_{v b 1}^{*} N_{v b 1} \beta_{b c}+\mu_{d}+\right.\right.} \\
& \left.\left.\left(I_{v a 1}^{*} N_{v a 1} \alpha_{a} \beta_{a c}+N_{v b 1} \alpha_{b} \beta_{b c}\right) \sin \varepsilon t\right)\left(1+\rho_{v b} \sin \varepsilon t\right)\right) \\
& I_{v a 1}^{*}=\left[\left(2 \left(-2 \lambda_{b i} \mu_{d}\left(\kappa_{c 1}+\mu_{d}\right) \mu_{v_{a}}\right.\right.\right. \\
& +2 N_{v a 1} \beta_{a c} \lambda_{v a l}\left(\kappa_{c 2}+\mu_{d}\right) \mu_{v_{b}} \\
& +N_{v a 1} \alpha_{a} \beta_{a c} \lambda_{v a 1}\left(\kappa_{c 2}+\mu_{d}\right) \\
& \mu_{v b} \rho_{v a}-N_{v b 1} \beta_{b c} \lambda_{b b 1}\left(\kappa_{c 1}+\mu_{d}\right) \mu_{v_{a}}\left(2+\alpha_{b} \rho_{v b}\right) \\
& \left.+N_{t c} N_{v a 1} \beta_{a c} \lambda_{v a 1} \lambda_{b b 1} \mu_{d}\left(2+\rho_{v a} \rho_{v b}+\alpha_{a}\left(\rho_{v a}+\rho_{v b}\right)\right)\right) \\
& -2\left(N_{v a 1} \alpha_{a} \beta_{a c} \lambda_{v a 1}\left(\kappa_{c 2}+\mu_{d}\right) \mu_{v i} \rho_{v a}\right. \\
& -N_{v b 1} \alpha_{b} \beta_{b c} \lambda_{v b 1}\left(\kappa_{c 1}+\mu_{d}\right) \mu_{v a} \rho_{v b} \\
& \left.+N_{t c} N_{v a 1} \beta_{a c} \lambda_{v a 1} \lambda_{v b 1} \mu_{d}\left(\rho_{v a} \rho_{v b}+\alpha_{a}\left(\rho_{v a}+\rho_{v b}\right)\right)\right) \cos \\
& 2 \varepsilon t+\left(4 \left(N_{v a 1} \beta_{a c} \lambda_{v a l}\left(\kappa_{c 2}+\mu_{d}\right) \mu_{v_{b}}\left(\alpha_{a}+\rho_{v a}\right)\right.\right. \\
& \left.-\lambda_{b b 1} \mu_{d}\left(\kappa_{c 1}+\mu_{d}\right) \mu_{v_{a}}-N_{v b 1} \beta_{b c} \lambda_{b b 1}\left(\kappa_{c 1}+\mu_{d}\right) \mu_{v_{a}}\left(\alpha_{b}+\rho_{v b}\right)\right) \\
& +N_{t c} N_{v a 1} \beta_{a c} \lambda_{v a 1} \lambda_{v b 1} \mu_{d}\left(4\left(\rho_{v a}+\rho_{v b}\right)\right. \\
& \left.\left.+\alpha_{a}\left(4+3 \rho_{v a} \rho_{v b}\right)\right)\right) \sin \varepsilon t \\
& \left.\left.-N_{t c} N_{v a 1} \alpha_{a} \beta_{a c} \lambda_{v a 1} \lambda_{v b 1} \mu_{d} \rho_{v a} \rho_{v b} \sin \varepsilon t\right)\right] \\
& /\left[\left(2 N _ { v a 1 } \beta _ { a c } ( 1 + \alpha _ { a } \operatorname { s i n } \varepsilon t ) \left(2 \lambda_{b b 1}\left(\kappa_{c 1}+\mu_{d}\right) \mu_{v_{a}}\right.\right.\right. \\
& +2 \lambda_{v a 1}\left(\kappa_{c 2}+\mu_{d}\right) \mu_{v_{b}}\left(\kappa_{c 2}+\mu_{d}\right) \mu_{v_{b}} \\
& +N_{t c} \mu_{d} \lambda_{v a l} \lambda_{v b 1}\left(2+\rho_{v a} \rho_{v b}\right)-N_{t c} \mu_{d} \lambda_{v a 1} \lambda_{v b 1} \rho_{v a} \rho_{v b} \cos 2 \varepsilon t \\
& +2\left(\lambda_{v a l}\left(\kappa_{c 2}+\mu_{d}\right) \mu_{v b} \rho_{v a}+\lambda_{v b 1}\left(\kappa_{c 1}+\mu_{d}\right) \mu_{v b} \rho_{v b}\right. \\
& \left.\left.\left.\left.+N_{t c} \mu_{d} \lambda_{v a l} \lambda_{b b 1}\left(\rho_{v a}+\rho_{v b}\right)\right) \sin \varepsilon t\right)\right)\right]
\end{aligned}
$$

$$
\begin{aligned}
& I_{a 2}^{*}=\left[\left(I _ { v b 2 } ^ { * } N _ { v b 2 } \beta _ { b a } ( 1 + \alpha _ { b } \operatorname { s i n } \varepsilon t ) \left(N_{t a} \lambda_{v b 2} \mu_{d}\right.\right.\right. \\
& \left.\left.+\left(\kappa_{a 2}+\mu_{d}\right) \mu_{v_{b}}+N_{t a} \lambda_{v b 2} \mu_{d} \rho_{v b} \sin \varepsilon t\right)\right) \\
& \text { / } N_{t a} \lambda_{v b 2}\left(\kappa_{a 2}+\mu_{d}\right)\left(I_{v a 2}^{*} N_{v a 2} \beta_{a a}\right. \\
& +I_{v b 2}^{*} N_{v b 2} \beta_{b a}+\mu_{d}+\left(I_{v a 2}^{*} N_{v a 2} \alpha_{a} \beta_{a a}\right. \\
& \left.\left.\left.\left.+N_{v b 2} \alpha_{b} \beta_{b a}\right) \sin \varepsilon t\right)\left(1+\rho_{v b} \sin \varepsilon t\right)\right)\right] \\
& I_{v a 2}^{*}=\left[\left(2 \left(-2 \lambda_{b 22} \mu_{d}\left(\kappa_{a 1}+\mu_{d}\right) \mu_{v_{a}}+2 N_{v a 2} \beta_{a a} \lambda_{v a 2}\left(\kappa_{a 2}+\mu_{d}\right) \mu_{v_{b}}\right.\right.\right. \\
& +N_{v a 2} \alpha_{a} \beta_{a a} \lambda_{v a 2}\left(\kappa_{a 2}+\mu_{d}\right) \mu_{v_{b}} \rho_{v a} \\
& -N_{v b 2} \beta_{b a} \lambda_{b b 2}\left(\kappa_{a 1}+\mu_{d}\right) \mu_{v a}\left(2+\alpha_{b} \rho_{v b}\right) \\
& \left.+N_{t a} N_{v a 2} \beta_{a a} \lambda_{v a 2} \lambda_{v b 2} \mu_{d}\left(2+\rho_{v a} \rho_{v b}+\alpha_{a}\left(\rho_{v a}+\rho_{v b}\right)\right)\right) \\
& -2\left(N_{v a 2} \alpha_{a} \beta_{a a} \lambda_{v a 2}\left(\kappa_{a 2}+\mu_{d}\right) \mu_{v b} \rho_{v a}\right. \\
& -N_{v b 2} \alpha_{b} \beta_{b a} \lambda_{v b 2}\left(\kappa_{a 1}+\mu_{d}\right) \mu_{v a} \rho_{v b} \\
& \left.+N_{t a} N_{v a 2} \beta_{a a} \lambda_{v a 2} \lambda_{b b 2} \mu_{d}\left(\rho_{v a} \rho_{v b}+\alpha_{a}\left(\rho_{v a}+\rho_{v b}\right)\right)\right) \cos 2 \varepsilon t \\
& +\left(4 \left(N_{v a 2} \beta_{a a} \lambda_{v a 2}\left(\kappa_{a 2}+\mu_{d}\right) \mu_{v_{b}}\left(\alpha_{a}+\rho_{v a}\right)\right.\right. \\
& \left.-\lambda_{b 22} \mu_{d}\left(\kappa_{a 1}+\mu_{d}\right) \mu_{v_{a}}-N_{v b 2} \beta_{b a} \lambda_{b 22}\left(\kappa_{a 1}+\mu_{d}\right) \mu_{v_{a}}\left(\alpha_{b}+\rho_{v b}\right)\right) \\
& +N_{t a} N_{v a 2} \beta_{a a} \lambda_{v a 2} \lambda_{b b 2} \mu_{d}\left(4\left(\rho_{v a}+\rho_{v b}\right)\right. \\
& \left.\left.+\alpha_{a}\left(4+3 \rho_{v a} \rho_{v b}\right)\right)\right) \sin \varepsilon t \\
& \left.\left.-N_{t a} N_{v a 2} \alpha_{a} \beta_{a a} \lambda_{v a 2} \lambda_{v b 2} \mu_{d} \rho_{v a} \rho_{v b} \sin \varepsilon t\right)\right] \\
& /\left[\left(2 N _ { v a 2 } \beta _ { a a } ( 1 + \alpha _ { a } \operatorname { s i n } \varepsilon t ) \left(2 \lambda_{v b 2}\left(\kappa_{a 1}+\mu_{d}\right) \mu_{v_{a}}\right.\right.\right. \\
& +2 \lambda_{v a 2}\left(\kappa_{a 2}+\mu_{d}\right) \mu_{v_{b}}\left(\kappa_{a 2}+\mu_{d}\right) \mu_{v_{b}}+N_{t a} \mu_{d} \lambda_{v a 2} \lambda_{v b 2}\left(2+\rho_{v a} \rho_{v b}\right) \\
& -N_{t a} \mu_{d} \lambda_{v a 2} \lambda_{b b 2} \rho_{v a} \rho_{v b} \cos 2 \varepsilon t+2\left(\lambda_{v a 2}\left(\kappa_{a 2}+\mu_{d}\right) \mu_{v b} \rho_{v a}\right. \\
& \left.\left.\left.\left.+\lambda_{b b 2}\left(\kappa_{a 1}+\mu_{d}\right) \mu_{v a} \rho_{v b}+N_{t a} \mu_{d} \lambda_{v a 2} \lambda_{b 2}\left(\rho_{v a}+\rho_{v b}\right)\right) \sin \varepsilon t\right)\right)\right]
\end{aligned}
$$




$$
\begin{aligned}
& I_{v b 2}^{*}=\left[\left(N_{t a} N_{v b 2} \beta_{a a} \lambda_{v b 2} \mu_{d}-\left(I_{v a 2}^{*} N_{v a 2} \beta_{a a}+\mu_{d}\right)\left(\kappa_{a 2}+\mu_{d}\right) \mu_{v_{b}}\right.\right. \\
& +\sin \varepsilon t\left(-I_{v a 2}^{*} N_{v a 2} \alpha_{a} \beta_{a a}\left(\kappa_{a 2}+\mu_{d}\right) \mu_{v_{b}}\right. \\
& +N_{t a} N_{v b 2} \beta_{b a} \lambda_{v b 2} \mu_{d}\left(\alpha_{b}+\rho_{v b}\right) \\
& \left.\left.\left.+N_{t a} N_{v b 2} \alpha_{b} \beta_{b a} \lambda_{v b 2} \mu_{d} \rho_{v b} \sin \varepsilon t\right)\right)\right] / \\
& {\left[N _ { v b 2 } \beta _ { b a } ( 1 + \alpha _ { b } \operatorname { s i n } \varepsilon t ) \left(N_{t a} \lambda_{v b 2} \mu_{d}+\left(\kappa_{a 2}+\mu_{d}\right) \mu_{v_{b}}\right.\right.} \\
& \left.\left.\left.+N_{t a} \lambda_{v b 2} \mu_{d} \rho_{v b} \sin \varepsilon t\right)\right)\right]
\end{aligned}
$$

\section{Local Asymptotically Stability}

The local stability of each equilibrium state is determined from Jacobian matrix of right hand side of the above set of differential equations evaluated at the equilibrium state.

Proposition A. If $S_{0}<1, S_{0 c}<1$ and $S_{0 a}<1$ when $\varepsilon=0$, then the disease free equilibrium state $E_{0 c}$ in children and $E_{0 a}$ in adult are locally asymptotically stable, where:

$$
\begin{aligned}
& S_{0}=\max \left\{\begin{array}{l}
2 N_{v b 1} \alpha_{b} \beta_{b c} \lambda_{v b 1}\left(\kappa_{c 1}+\mu_{d}\right) \mu_{v_{a}} \rho_{v b}+ \\
N_{v a 1} \beta_{a c} \lambda_{v a 1}\left(2\left(N_{t c} \lambda_{v b 1} \mu_{d}+\left(\kappa_{c 2}+\mu_{d}\right) \mu_{v_{b}}\right)\right. \\
\frac{\left.\left(2+\alpha_{a} \rho_{v a}\right)+2 N_{t c} \lambda_{v b 1} \mu_{d}\left(\alpha_{a}+\rho_{v a}\right)\right)}{\left(2 \lambda_{v b 1}\left(\kappa_{c 1}+\mu_{d}\right) \mu_{v_{a}}\left(2 \mu_{d}+N_{v b 1} \beta_{b c}\left(2+\alpha_{b} \rho_{v b}\right)\right)\right.} \\
+2 N_{v a 1} \beta_{a c} \lambda_{v a 1}\left(\alpha_{a}\left(\kappa_{c 2}+\mu_{d}\right) \mu_{v_{b}} \rho_{v a}\right. \\
\left.\left.+N_{t c} \lambda_{v b 1} \mu_{d}\left(\alpha_{a} \rho_{v a}+\left(\alpha_{a}+\rho_{v a}\right) \rho_{v b}\right)\right)\right) \\
2 N_{v b 2} \alpha_{b} \beta_{b a} \lambda_{v b 2}\left(\kappa_{a 1}+\mu_{d}\right) \mu_{v_{a}} \rho_{v b} \\
+N_{v a 2} \beta_{a a} \lambda_{v a 2}\left(2\left(N_{t a} \lambda_{v b 2} \mu_{d}+\left(\kappa_{a 2}+\mu_{d}\right) \mu_{v_{b}}\right)\right. \\
\frac{\left.\left(2+\alpha_{a} \rho_{v a}\right)+2 N_{t a} \lambda_{v b 2} \mu_{d}\left(\alpha_{a}+\rho_{v a}\right)\right)}{\left(2 \lambda _ { v b 2 } ( \kappa _ { a 1 } + \mu _ { d } ) \mu _ { v _ { a } } \left(2 \mu_{d}+N_{v b 2} \beta_{b a}\right.\right.} \\
\left.\left(2+\alpha_{b} \rho_{v b}\right)\right)+2 N_{v a 2} \beta_{a a} \lambda_{v a 2}\left(\alpha_{a}\left(\kappa_{a 2}+\mu_{d}\right)\right. \\
\left.\left.\mu_{v_{b}} \rho_{v a}+N_{t a} \lambda_{v b 2} \mu_{d}\left(\alpha_{a} \rho_{v a}+\left(\alpha_{a}+\rho_{v a}\right) \rho_{v b}\right)\right)\right)
\end{array}\right\} \\
& 2 N_{v b 1} \alpha_{b} \beta_{b c} \lambda_{v b 1}\left(\kappa_{c 1}+\mu_{d}\right) \mu_{v_{a}} \rho_{v b} \\
& +N_{v a 1} \beta_{a c} \lambda_{v a 1}\left(2\left(N_{t c} \lambda_{v b 1} \mu_{d}+\left(\kappa_{c 2}+\mu_{d}\right) \mu_{v_{b}}\right)\right. \\
& S_{0 c}=\frac{\left.\left(2+\alpha_{a} \rho_{v a}\right)+2 N_{t c} \lambda_{v b 1} \mu_{d}\left(\alpha_{a}+\rho_{v a}\right)\right)}{\left(2 \lambda_{v b 1}\left(\kappa_{c 1}+\mu_{d}\right) \mu_{v_{a}}\left(2 \mu_{d}+N_{v b 1} \beta_{b c}\left(2+\alpha_{b} \rho_{v b}\right)\right)\right.} \\
& +2 N_{v a 1} \beta_{a c} \lambda_{v a 1}\left(\alpha_{a}\left(\kappa_{c 2}+\mu_{d}\right) \mu_{v_{b}} \rho_{v a}\right. \\
& \left.\left.+N_{t c} \lambda_{v b 1} \mu_{d}\left(\alpha_{a} \rho_{v a}+\left(\alpha_{a}+\rho_{v a}\right) \rho_{v b}\right)\right)\right) \\
& \text { in children } \\
& 2 N_{v b 2} \alpha_{b} \beta_{b a} \lambda_{v b 2}\left(\kappa_{a 1}+\mu_{d}\right) \mu_{v_{a}} \rho_{v b} \\
& +N_{v a 2} \beta_{a a} \lambda_{v a 2}\left(2 \left(N_{t a} \lambda_{v b 2} \mu_{d}+\left(\kappa_{a 2}\right.\right.\right. \\
& S_{0 a}=\frac{\left.\left.\left.+\mu_{d}\right) \mu_{v_{b}}\right)\left(2+\alpha_{a} \rho_{v a}\right)+2 N_{t a} \lambda_{v b 2} \mu_{d}\left(\alpha_{a}+\rho_{v a}\right)\right)}{\left(2 \lambda_{v b 2}\left(\kappa_{a 1}+\mu_{d}\right) \mu_{v_{a}}\left(2 \mu_{d}+N_{v b 2} \beta_{b a}\left(2+\alpha_{b} \rho_{v b}\right)\right)\right.} \text { in adult } \\
& +2 N_{v a 2} \beta_{a a} \lambda_{v a 2}\left(\alpha_{a}\left(\kappa_{a 2}+\mu_{d}\right) \mu_{v_{b}} \rho_{v a}\right. \\
& \left.\left.+N_{t a} \lambda_{v b 2} \mu_{d}\left(\alpha_{a} \rho_{v a}+\left(\alpha_{a}+\rho_{v a}\right) \rho_{v b}\right)\right)\right)
\end{aligned}
$$

\section{Proof.}

For the disease free equilibrium state in children $\mathrm{E}_{0 \mathrm{c}}$ $=(1,0,0,0,0)$ and in adult $\mathrm{E}_{0 \mathrm{a}}=(1,0,0,0,0)$.
The system defined by Equation (4a) - (4j), the Jacobian matrix evaluated at $\mathrm{E}_{0 \mathrm{c}}$ and $\mathrm{E}_{0 \mathrm{a}}$ respectively, given by:

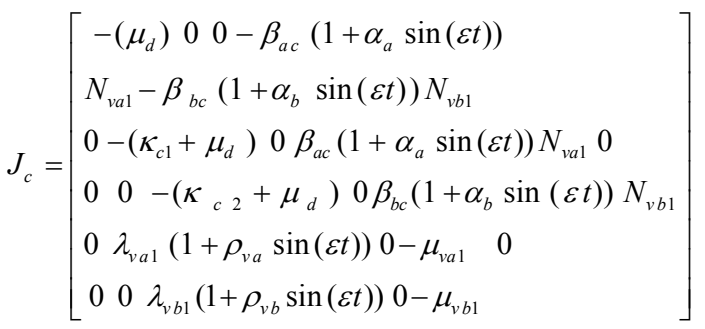

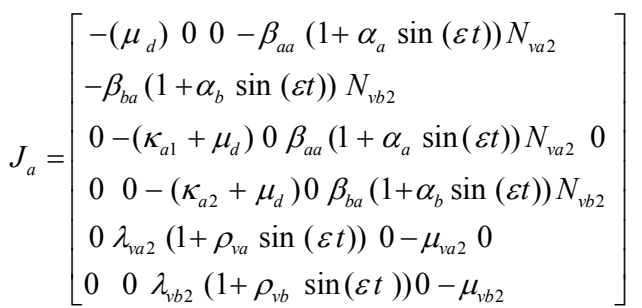

The eigenvalues are obtained by solving the characteristic equations, det $\left|\eta \mathrm{I}_{5}-\mathrm{J}_{\mathrm{i}}\right|=0$. Where $I_{5}$ is the $5 \times 5$ identity matrix and $\mathrm{J}_{\mathrm{i}}\left(\mathrm{J}_{\mathrm{i}} ; \mathrm{i}=\mathrm{c}, \mathrm{a}\right)$ is the Jacobian matrix matrix for (7a) and (7b), respectively. To evaluate the determinant, we get the following characteristic equations:

$$
\begin{aligned}
& \left(\eta+\mu_{d}\right)\left(\eta^{4}+W_{1} \eta^{3}+W_{2} \eta^{2}+W_{3} \eta+W_{4}\right)=0 \\
& \left(\eta+\mu_{d}\right)\left(\eta^{4}+F_{1} \eta^{3}+F_{2} \eta^{2}+F_{3} \eta+F_{4}\right)=0
\end{aligned}
$$

Where:

$$
\begin{aligned}
& W_{1}=\kappa_{c 1}+\kappa_{c 2}+2 \mu_{d}+\mu_{v_{a}}+\mu_{v_{b}} \\
& \left.W_{2}=-N_{v a 1} \beta_{a c} \lambda_{v a 1}-N_{v b 1} \beta_{b c} \lambda_{v b 1}\right) \\
& +\mu_{d}^{2}+2 \mu_{d} \mu_{v_{a}}+2 \mu_{d} \mu_{v_{b}}+\mu_{v_{a}} \mu_{v_{b}} \\
& +\kappa_{c 2}\left(\mu_{d}+\mu_{v_{a}}+\mu_{v_{b}}\right)+\kappa_{c 1}\left(\kappa_{c 2}+\mu_{d}+\mu_{v_{a}}+\mu_{v_{b}}\right) \\
& W_{3}=\left(\kappa_{c 1}+\mu_{d}\right)\left(\kappa_{c 2}+\mu_{d}\right) \mu_{v_{a}}-N_{v b 1} \beta_{b c} \lambda_{v b 1}\left(\kappa_{c 1}+\mu_{d}+\mu_{v_{a}}\right) \\
& +\left(\left(\kappa_{c 1}+\mu_{d}\right)\left(\kappa_{c 2}+\mu_{d}\right)+\left(\kappa_{c 1}+\kappa_{c 2}+2 \mu_{d}\right) \mu_{v_{a}}\right) \\
& \mu_{v_{b}}-N_{v a 1} \beta_{a c} \lambda_{v a 1}\left(\kappa_{c 2}+\mu_{d}+\mu_{v_{b}}\right)+\left(\beta_{a c} \lambda_{v a 1}\left(\kappa_{c 2}+\mu_{d}+\mu_{v_{b}}\right)\right) \\
& W_{4}=\left(N_{v a 1} \beta_{a c} \lambda_{v a 1}-\left(\kappa_{c 1}+\mu_{d}\right) \mu_{v_{a}}\right) \\
& \quad\left(N_{v b 1} \beta_{b c} \lambda_{v b 1}+\left(\kappa_{c 2}+\mu_{d}\right) \mu_{v_{b}}\right) \\
& F_{1}=\kappa_{a 1}+\kappa_{a 2}+2 \mu_{d}+\mu_{v_{a}}+\mu_{v_{b}} \\
& \left.F_{2}=-N_{v a 2} \beta_{a a} \lambda_{v a 2}-N_{v b 2} \beta_{b a} \lambda_{v b 2}\right)+\mu_{d}^{2}+2 \mu_{d} \mu_{v_{a}}+2 \mu_{d} \mu_{v_{b}} \\
& +\mu_{v_{a}} \mu_{v_{b}}+\kappa_{a 2}\left(\mu_{d}+\mu_{v_{a}}+\mu_{v_{b}}\right)+\kappa_{a 1}\left(\kappa_{a 2}+\mu_{d}+\mu_{v_{a}}+\mu_{v_{b}}\right)
\end{aligned}
$$




$$
\begin{aligned}
& F_{3}=\left(\kappa_{a 1}+\mu_{d}\right)\left(\kappa_{a 2}+\mu_{d}\right) \mu_{v_{a}}-N_{v b 2} \beta_{b a} \lambda_{v b 2}\left(\kappa_{a 1}+\mu_{d}+\mu_{v_{a}}\right) \\
& +\left(\left(\kappa_{a 1}+\mu_{d}\right)\left(\kappa_{a 2}+\mu_{d}\right)+\left(\kappa_{a 1}+\kappa_{a 2}+2 \mu_{d}\right) \mu_{v_{a}}\right) \mu_{v_{b}} \\
& -N_{v a 2} \beta_{a a} \lambda_{v a 2}\left(\kappa_{a 2}+\mu_{d}+\mu_{v_{b}}\right)+\left(\beta_{a a} \lambda_{v a 2}\left(\kappa_{a 2}+\mu_{d}+\mu_{v_{b}}\right)\right) \\
& F_{4}=\left(N_{v a 2} \beta_{a a} \lambda_{v a 2}-\left(\kappa_{a 1}+\mu_{d}\right) \mu_{v_{a}}\right)\left(N_{v b 2} \beta_{b a} \lambda_{v b 2}+\left(\kappa_{a 2}+\mu_{d}\right) \mu_{v_{b}}\right)
\end{aligned}
$$

From the characteristic equation, Equation (8a)-(8b), we see that eigenvalues are $\eta_{c}=-\mu_{d}$ and $\eta_{a}=-\mu_{d}$, all of these eigenvalues are negative, for $S_{0}<1$. The sign of other four eigenvalues can be ascertained by solving equation $\left(\eta^{4}+W_{1} \eta^{3}+W_{2} \eta^{2}+W_{3} \eta+W_{4}\right)=0$ and $\left(\eta^{4}+F_{1} \eta^{3}+F_{2} \eta^{2}+F_{3} \eta+F_{4}\right)=0$. The remaining four eigenvalues have negative real parts if they satisfy Routh-Hurwitz criteria (10a) - (10d) (Esteva and Vargas,
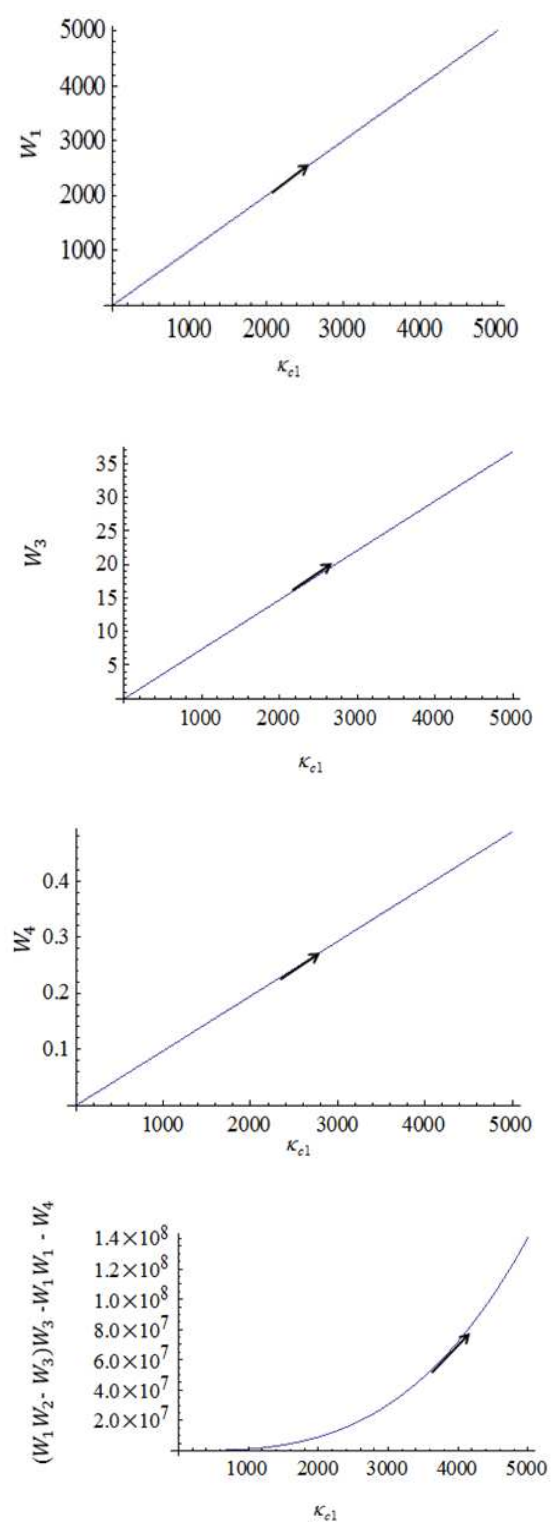

1998), each equilibrium state is locally asymptotically stable if the following conditions are satisfied:

$W_{1}$ and $F_{1}>0$

$W_{3}$ and $F_{3}>0$

$W_{4}$ and $F_{4}>0$

$\left(W_{1} W_{2}-W_{3}\right) W_{3}+W_{1}^{2} W_{4}>0$ and $\left(F_{1} F_{2}-F_{3}\right) F_{3}+F_{1}^{2} F_{4}>0$

After we use Mathematica to show the conditions of locally asymptotically stable, we can see that $W_{l}$ and $F_{l}$ are always positive. For the equations given by (10b)-(10d), we show these conditions by using the following Fig. 2.
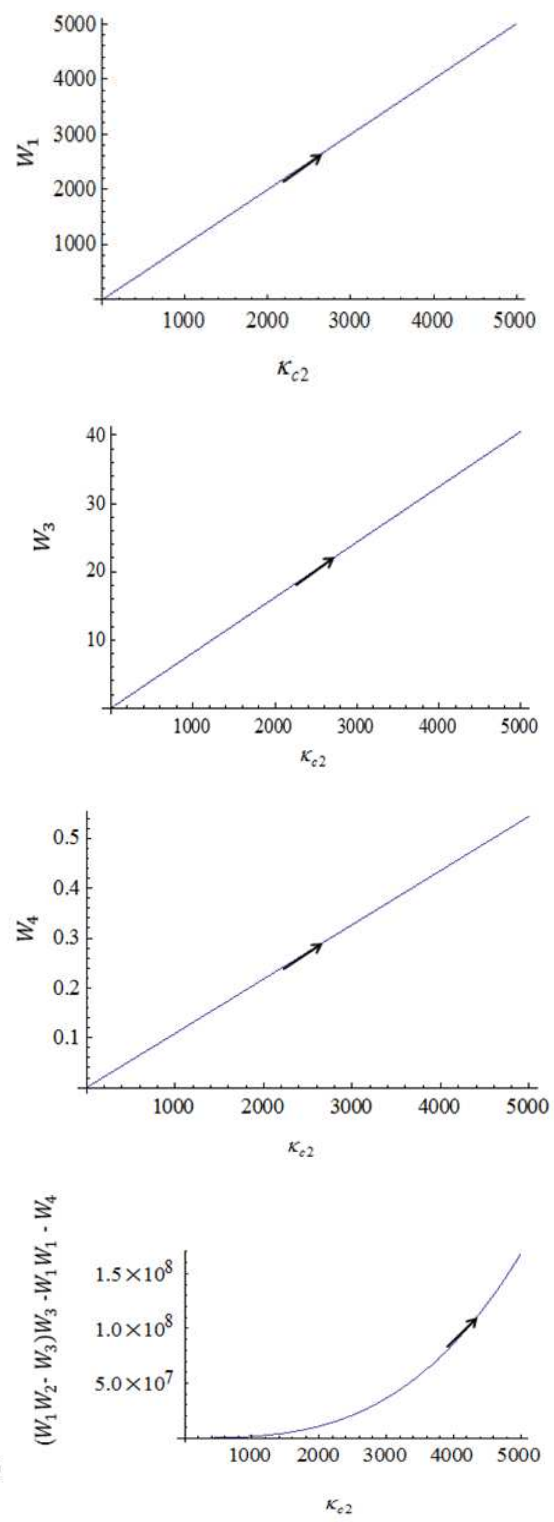

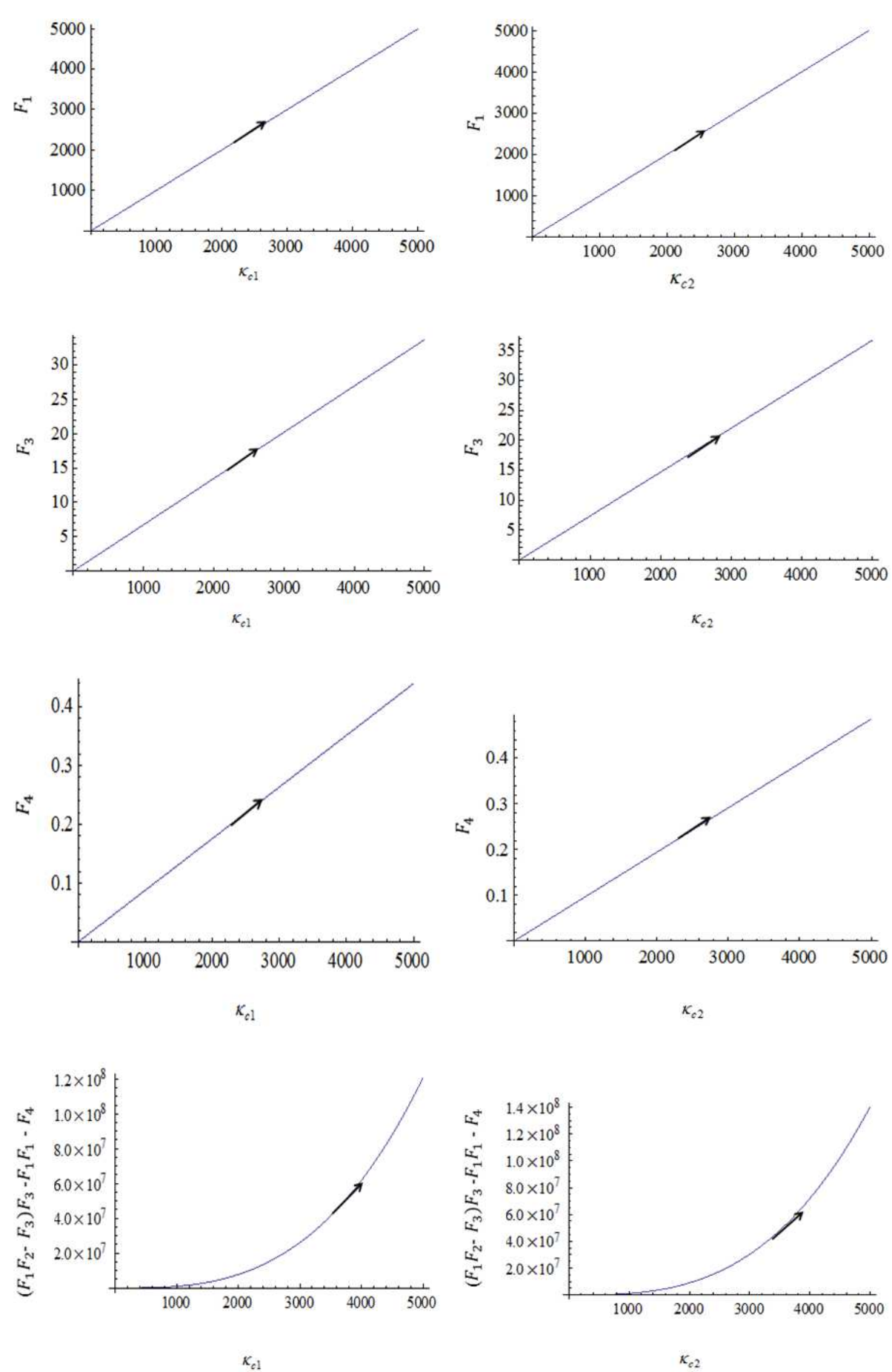

Fig. 2. The parameter spaces for the disease free equilibrium state, which satisfies the Routh-Hurwitz conditions, show onto $\left(\kappa_{c 1}, W_{3}\right),\left(\kappa_{c 2}, W_{3}\right),\left(\kappa_{a 1}, F_{3}\right),\left(\kappa_{a 2}, F_{3}\right),\left(\kappa_{c 1}, W_{4}\right),\left(\kappa_{c 2}, W_{4}\right),\left(\kappa_{a 1}, F_{4}\right),\left(\kappa_{a 2}, F_{4}\right),\left(\kappa_{c 1},\left(\left(W_{1} W_{2}-W_{3}\right) W_{3}-W_{1}^{2} W_{4}\right)\right)$, $\left(\kappa_{c 1},\left(\left(W_{1} W_{2}-W_{3}\right) W_{3}-W_{1}^{2} W_{4}\right)\right),\left(\kappa_{a 1},\left(\left(F_{1} F_{2}-F_{3}\right) F_{3}-F_{1}^{2} F_{4}\right)\right),\left(\kappa_{a 2},\left(\left(F_{1} F_{2}-F_{3}\right) F_{3}-F_{1}^{2} F_{4}\right)\right)$, respectively. The values of parameter are follows: $\kappa_{c 1}=1 /(17 / 2), \quad \kappa_{c 2}=1 /(19 / 2), \quad \mu_{d}=1 /\left(365^{*} 74.6\right)$ day $^{-1}, N_{t c}=9000, \quad N_{v a 1}=4000, \quad N_{v b 1}=5500, \quad \beta_{a c}=0.00769$, $\beta_{b c}=0.000246, \lambda_{v a 1}=0.00000576, \lambda_{v b 1}=0.00000335, \quad \alpha_{a}=0.07, \quad \alpha_{b}=0.067$ and $N_{t}=100000, \kappa_{a 1}=1 /(19 / 2), \kappa_{a 2}=1 /(21 / 2)$, $\mu_{d}=1 /(365 * 74.6)$ day $^{-1}, N_{t a}=6000, N_{v a 2}=3000, N_{v b 2}=4100, \beta_{a a}=0.000045, \beta_{b a}=0.000067, \lambda_{v a 2}=0.0066, \lambda_{v b 2}=0.00235$, $\alpha_{a}=0.07, \alpha_{b}=0.067$ and $N_{t}=100000$. From the above figures, the Routh-Hurwitz conditions are satisfied for $S_{0}>1$ 


\section{Endemic Disease State}

Proposition B. If $\mathrm{S}_{0}>1$, when $\varepsilon=0$, then the equilibrium state $\quad \hat{S}=\left(S_{c}^{*}, I_{c 1}^{*}, I_{c 2}^{*}, I_{v a 1}^{*}, I_{v b 1}^{*}, S_{a}^{*}, I_{a 1}^{*}, I_{a 2}^{*}, I_{v a 2}^{*}, I_{v b 2}^{*}\right) \quad$ is locally asymptotically stable.

\section{Proof.}

For the endemic disease equilibrium state $E_{1 c}=\left(S_{c}^{*}, I_{c 1}^{*}, I_{c 2}^{*}, I_{v a 1}^{*}, I_{v b 1}^{*}\right)$ in children and $E_{1 a}=\left(S_{a}^{*}, I_{a 1}^{*}, I_{a 2}^{*}, I_{v a 2}^{*}, I_{v b 2}^{*}\right)$ in adult, we obtain the characteristic equation:

$$
\begin{aligned}
& \left(\eta^{5}+D_{1} \eta^{4}+D_{2} \eta^{3}+D_{3} \eta^{2}+D_{4} \eta+D_{5}\right)=0 \text { in children } \\
& \left(\eta^{5}+G_{1} \eta^{4}+G_{2} \eta^{3}+G_{3} \eta^{2}+G_{4} \eta+G_{5}\right)=0 \text { in adult }
\end{aligned}
$$

Where:

$$
\begin{aligned}
& D_{1}=N_{v a 1} \beta_{a c} \theta_{1}+N_{v b 1} \beta_{b c} \theta_{2}+\kappa_{c 1}+\kappa_{c 2}+3 \mu_{d}+\mu_{v_{a}}+\mu_{v_{b}} \\
& D_{2}=\kappa_{c 1} \kappa_{c 2}+2 \kappa_{c 1} \mu_{d}+2 \kappa_{c 2} \mu_{d}+3 \mu_{d}^{2}+\kappa_{c 1} \mu_{v_{a}}+\kappa_{c 2} \mu_{v a 1} \\
& +3 \mu_{d} \mu_{v_{a}}\left(\kappa_{c 1}+\kappa_{c 2}+3 \mu_{d}+\mu_{v a 1}\right) \mu_{v_{b}}+N_{v a 1} \beta_{a c} \\
& \left(-\left(\lambda_{v a 1} \mu_{d} / N_{v a 1} \beta_{a c} \theta_{1}+N_{v b 1} \beta_{b c} \theta_{2} \mu_{d}\right)+\theta_{1}\left(\kappa_{c 1}+\kappa_{c 2}+2 \mu_{d}\right.\right. \\
& \left.\left.+\left(\lambda_{v a 1} \mu_{d} / N_{v a 1} \beta_{a c} \theta_{1}+N_{v b 1} \beta_{b c} \theta_{2} \mu_{d}\right)+\mu_{v_{a}}+\mu_{v_{b}}\right)\right) \\
& +N_{v b 1} \beta_{b c}\left(-\left(\lambda_{v b 1} \mu_{d} / N_{v a 1} \beta_{a c} \theta_{1}+N_{v b 1} \beta_{b c} \theta_{2} \mu_{d}\right)+\theta_{2}\left(\kappa_{c 1}\right.\right. \\
& \left.\left.+\kappa_{c 2}+2 \mu_{d}+\left(\lambda_{v b 1} \mu_{d} / N_{v a 1} \beta_{a c} \theta_{1}+N_{v b 1} \beta_{b c} \theta_{2} \mu_{d}\right) \mu_{v_{a}}+\mu_{v_{b}}\right)\right)
\end{aligned}
$$

$$
\begin{aligned}
& D_{4}=\kappa_{c 1} \kappa_{c 2} \mu_{d} \mu_{v_{a}}+\kappa_{c 1} \mu_{d}^{2} \mu_{v_{a}}+\kappa_{c 2} \mu_{d}^{2} \mu_{v_{a}}+\mu_{d}^{3} \mu_{v_{a}}+\kappa_{c 1} \kappa_{c 2} \mu_{d} \mu_{v_{b}} \\
& +\kappa_{c 1} \mu_{d}^{2} \mu_{v_{b}}+\kappa_{c 2} \mu_{d}^{2} \mu_{v_{b}}+\mu_{d}^{3} \mu_{v_{b}}+\kappa_{c 1} \kappa_{c 2} \mu_{v_{a}} \mu_{v_{b}}+2 \kappa_{c 1} \mu_{d} \mu_{v_{a}} \mu_{v_{b}} \\
& +2 \kappa_{c 2} \mu_{d} \mu_{v_{a}} \mu_{v_{b}}+3 \mu_{d}^{3} \mu_{v_{a}} \mu_{v_{b}}+\frac{1}{N_{v a 1} \beta_{a c} \theta_{1}+N_{v b 1} \beta_{b c} \theta_{2}+\mu_{d}} \\
& N_{v b 1} \beta_{b c}\left(\left(-1+\theta_{2}\right) \lambda_{v b 1} \mu_{d}^{2}\left(\mu_{d}+2 \mu_{v_{a}}\right)+\theta_{2}\left(N_{v a 1} \beta_{a c} \theta_{1}+N_{v b 1} \beta_{b c} \theta_{2}+\mu_{d}\right)\right. \\
& \left(\mu_{d}\left(\kappa_{c 2}+\mu_{d}\right) \mu_{v_{a}}+\left(\mu_{d}\left(\kappa_{c 2}+\mu_{d}\right)+\left(\kappa_{c 2}+2 \mu_{d}\right) \mu_{v_{a}}\right) \mu_{v_{b}}\right) \\
& +\kappa_{c 1}\left(\left(-1+\theta_{2}\right) \lambda_{v b 1} \mu_{d}\left(\mu_{d}+2 \mu_{v_{a}}\right)+\theta_{2}\left(N_{v a 1} \beta_{a c} \theta_{1}+N_{v b 1} \beta_{b c} \theta_{2}+\mu_{d}\right)\left(\left(\kappa_{c 2}+\mu_{d}\right) \mu_{v_{a}}\right.\right. \\
& \left.\left.\left.+\left(\kappa_{c 2}+\mu_{d}+\mu_{v_{a}}\right) \mu_{v_{b}}\right)\right)\right)+N_{v a 1} \beta_{a c}\left(-\frac{\kappa_{c 2} \lambda_{v a 1} \mu_{d}^{2}}{N_{v a 1} \beta_{a c} \theta_{1}+N_{v b 1} \beta_{b c} \theta_{2}+\mu_{d}}+\frac{\theta_{1} \kappa_{c 2} \lambda_{v a 1} \mu_{d}^{2}}{N_{v a 1} \beta_{a c} \theta_{1}+N_{v b 1} \beta_{b c} \theta_{2}+\mu_{d}}\right. \\
& -\frac{\lambda_{v a 1} \mu_{d}^{3}}{N_{v a 1} \beta_{a c} \theta_{1}+N_{v b 1} \beta_{b c} \theta_{2}+\mu_{d}}+\frac{\theta_{1} \lambda_{v a 1} \mu_{d}^{3}}{N_{v a 1} \beta_{a c} \theta_{1}+N_{v b 1} \beta_{b c} \theta_{2}+\mu_{d}}+\theta_{1} \kappa_{c 1} \kappa_{c 2} \mu_{v_{a}}+\theta_{1} \kappa_{c 1} \mu_{d} \mu_{v_{a}} \\
& +\theta_{1} \kappa_{c 2} \mu_{d} \mu_{v_{a}}+\theta_{1} \mu_{d}^{2} \mu_{v_{a}}+\theta_{1} \kappa_{c 1} \kappa_{c 2} \mu_{v_{b}}+\theta_{1} \kappa_{c 1} \mu_{d} \mu_{v_{b}}+\theta_{1} \kappa_{c 2} \mu_{d} \mu_{v_{b}}+\theta_{1} \mu_{d}^{2} \mu_{v_{b}} \\
& -\frac{\kappa_{c 2} \lambda_{v a 1} \mu_{d} \mu_{v_{b}}}{N_{v a 1} \beta_{a c} \theta_{1}+N_{v b 1} \beta_{b c} \theta_{2}+\mu_{d}}+\frac{\theta_{1} \kappa_{c 2} \lambda_{v a 1} \mu_{d} \mu_{v_{b}}}{N_{v a 1} \beta_{a c} \theta_{1}+N_{v b 1} \beta_{b c} \theta_{2}+\mu_{d}}-\frac{2 \lambda_{v a 1} \mu_{d}^{2} \mu_{v_{b}}}{N_{v a 1} \beta_{a c} \theta_{1}+N_{v b 1} \beta_{b c} \theta_{2}+\mu_{d}}+\frac{2 \theta_{1} \lambda_{v a 1} \mu_{d}^{2} \mu_{v_{b}}}{N_{v a 1} \beta_{a c} \theta_{1}+N_{v b 1} \beta_{b c} \theta_{2}+\mu_{d}} \\
& +\theta_{1} \kappa_{c 1} \mu_{v_{a}} \mu_{v_{b}}+\theta_{1} \kappa_{c 2} \mu_{v_{a}} \mu_{v_{b}}+2 \theta_{1} \mu_{d} \mu_{v_{a}} \mu_{v_{b}}+\frac{1}{\left(N_{v a 1} \beta_{a c} \theta_{1}+N_{v b 1} \beta_{b c} \theta_{2}+\mu_{d}\right)^{2}} N_{v b 1} \beta_{b c} \mu_{d}\left(N_{v a 1} \beta_{a c} \theta_{1}\right. \\
& \left(-\theta_{1} \lambda_{v b 1}\left(\kappa_{c 1}+\mu_{d}+\mu_{v_{a}}\right)+\theta_{2}\left(\left(-1+\theta_{1}\right) \kappa_{c 2} \lambda_{v a .1}-\lambda_{v a 1}\left(\mu_{d}+\mu_{v_{b}}\right)+\theta_{1}\left(\lambda_{v b 1}\left(\kappa_{c 1}+\mu_{d}+\mu_{v_{a}}\right)+\lambda_{v a 1}\left(\mu_{d}+\mu_{v_{b}}\right)\right)\right)\right) \\
& +N_{v b 1} \beta_{b c} \theta_{2}\left(-\theta_{1} \lambda_{v b 1}\left(\kappa_{c 1}+\mu_{d}+\mu_{v_{a}}\right)+\theta_{2}\left(\left(-1+\theta_{1}\right) \kappa_{c 2} \lambda_{v a 1}-\lambda_{v a 1}\left(\mu_{d}+\mu_{v b 1}\right)+\theta_{1}\left(\lambda_{v b 1}\left(\kappa_{c 1}+\mu_{d}+\mu_{v_{a}}\right)\right.\right.\right. \\
& \left.\left.\left.+\lambda_{v a 1}\left(\mu_{d}+\mu_{v_{b}}\right)\right)\right)\right)+\mu_{d}\left(-\lambda_{v b 1}\left(-\lambda_{v a 1}+\theta_{1}\left(\kappa_{c 1}+\lambda_{v a 1}+\mu_{d}+\mu_{v_{a}}\right)\right)+\theta_{2}\left(\left(-1+\theta_{1}\right) \kappa_{c 2} \lambda_{v a 1}-\lambda_{v a 1}\left(\lambda_{v b 1}+\mu_{d}+\mu_{v_{b}}\right)+\right.\right. \\
& \left.\left.\left.\left.\theta_{1}\left(\kappa_{c 1} \lambda_{v b 1}+\lambda_{v b 1}\left(\mu_{d}+\mu_{v_{a}}\right)+\lambda_{v a 1}\left(\lambda_{v b 1}+\mu_{d}+\mu_{v_{b}}\right)\right)\right)\right)\right)\right)
\end{aligned}
$$




$$
\begin{aligned}
& D_{5}=\frac{1}{\left(N_{v a 1} \beta_{a c} \theta_{1}+N_{v b 1} \beta_{b c} \theta_{2}+\mu_{d}\right)^{2}}\left(N_{v a 1}^{3} \beta_{a c}^{3} \theta_{1}^{3}\left(\kappa_{c 1}+\mu_{d}\right)\left(\kappa_{c 2}+\mu_{d}\right) \mu_{v a 1} \mu_{v_{b}}\right. \\
& +\left(N_{v b 1} \beta_{b c} \theta_{2}+\mu_{d}\right)\left(\kappa_{c 1}+\mu_{d}\right) \mu_{v_{a}}\left(N_{v b 1} \beta_{b c}\left(-1+\theta_{2}\right) \lambda_{v b 1} \mu_{d}^{2}+\left(N_{v b 1} \beta_{b c} \theta_{2}+\mu_{d}\right)^{2}\left(\kappa_{c 2}+\mu_{d}\right) \mu_{v_{b}}\right) \\
& +N_{v a 1}^{2} \beta_{a c}^{2} \theta_{1}\left(N_{v b 1} \beta_{b c} \theta_{1}\left(-1+\theta_{2}\right) \lambda_{v b 1} \mu_{d}\left(\kappa_{c 1}+\mu_{d}\right) \mu_{v_{a}}+\left(N_{v b 1} \beta_{b c} \theta_{2}+\mu_{d}\right)\left(\kappa_{c 2}+\mu_{d}\right)\right. \\
& \left.\left(\left(-1+\theta_{1}\right) \lambda_{v a 1} \mu_{d}+3 \theta_{1}\left(\kappa_{c 1}+\mu_{d}\right) \mu_{v_{a}}\right) \mu_{v_{b}}\right)+N_{v a 1} \beta_{a c}\left(\mu _ { d } ^ { 2 } ( \kappa _ { c 2 } + \mu _ { d } ) \left(\left(-1+\theta_{1}\right) \lambda_{v a 1} \mu_{d}\right.\right. \\
& \left.\left.+3 \theta_{1}\left(\kappa_{c 1}+\mu_{d}\right) \mu_{v_{a}}\right) \mu_{v_{b}}\right)+N_{v b 1}^{2} \beta_{b c}^{2} \theta_{2}\left(\theta_{1}\left(-1+\theta_{2}\right) \lambda_{v b 1} \mu_{d}\left(\kappa_{c 1}+\mu_{d}\right) \mu_{v_{a}}+\theta_{2}\left(\kappa_{c 2}+\mu_{d}\right)\left(\left(-1+\theta_{1}\right) \lambda_{v a 1} \mu_{d}+3 \theta_{1}\left(\kappa_{c 1}+\mu_{d}\right) \mu_{v_{a}}\right) \mu_{v_{b}}\right) \\
& \left.\left.\left.+N_{v b 1} \beta_{b c} \mu_{d}\left(\left(-1+\theta_{2}\right) \lambda_{v b 1} \mu_{d}+2 \theta_{1}\left(\kappa_{c 1}+\mu_{d}\right) \mu_{v_{a}}\right)+2 \theta_{2}\left(\kappa_{c 2}+\mu_{d}\right)\left(\left(-1+\theta_{1}\right) \lambda_{v a 1} \mu_{d}+3 \theta_{1}\left(\kappa_{c 1}+\mu_{d}\right) \mu_{v_{a}}\right) \mu_{v_{b}}\right)\right)\right) \\
& G_{1}=N_{v a 2} \beta_{a a} \theta_{3}+N_{v b 2} \beta_{b a} \theta_{4}+\kappa_{a 1}+\kappa_{a 2}+3 \mu_{d}+\mu_{v_{a}}+\mu_{v_{b}} \\
& G_{2}=\kappa_{a 1} \kappa_{a 2}+2 \kappa_{a 1} \mu_{d}+2 \kappa_{a 2} \mu_{d}+3 \mu_{d}^{2}+\kappa_{a 1} \mu_{v_{a}}+\kappa_{a 2} \mu_{v_{a}}+3 \mu_{d} \mu_{v_{a}}+ \\
& \left(\kappa_{a 1}+\kappa_{a 2}+3 \mu_{d}+\mu_{v_{a}}\right) \mu_{v_{b}}+N_{v a 2} \beta_{a a}\left(-\left(\lambda_{v a 2} \mu_{d} / N_{v a 2} \beta_{a a} \theta_{3}+N_{v b 2} \beta_{b a} \theta_{4} \mu_{d}\right)\right. \\
& \left.+\theta_{3}\left(\kappa_{a 1}+\kappa_{a 2}+2 \mu_{d}+\left(\lambda_{v a 2} \mu_{d} / N_{v a 2} \beta_{a a} \theta_{3}+N_{v b 2} \beta_{b a} \theta_{4} \mu_{d}\right)+\mu_{v_{a}}+\mu_{v_{b}}\right)\right)+N_{v b 2} \beta_{b a} \\
& \left(-\left(\lambda_{v b 2} \mu_{d} / N_{v a 2} \beta_{a a} \theta_{3}+N_{v b 2} \beta_{b a} \theta_{4} \mu_{d}\right)+\theta_{4}\left(\kappa_{a 1}+\kappa_{a 2}+2 \mu_{d}+\left(\lambda_{v b 2} \mu_{d} / N_{v a 2} \beta_{a a} \theta_{3}+N_{v b 2} \beta_{b a} \theta_{4} \mu_{d}\right) \mu_{v_{a}}+\mu_{v_{b}}\right)\right)
\end{aligned}
$$

$\left.\left.\left.+N_{v b 2} \beta_{b a} \theta_{4}+\mu_{d}\right)\left(\left(\kappa_{a 2}+\mu_{d}\right) \mu_{v_{a}}+\left(\kappa_{a 2}+\mu_{d}+\mu_{v_{a}}\right) \mu_{v_{b}}\right)\right)\right)+N_{v a 2} \beta_{a a}$

$\left(-\frac{\kappa_{a 2} \lambda_{v a 2} \mu_{d}^{2}}{N_{v a 2} \beta_{a a} \theta_{3}+N_{v b 2} \beta_{b a} \theta_{4}+\mu_{d}}+\frac{\theta_{3} \kappa_{a 2} \lambda_{v a 2} \mu_{d}^{2}}{N_{v a 2} \beta_{a a} \theta_{3}+N_{v b 2} \beta_{b a} \theta_{4}+\mu_{d}}-\frac{\lambda_{v a 2} \mu_{d}^{3}}{N_{v a 2} \beta_{a a} \theta_{3}+N_{v b 2} \beta_{b a} \theta_{4}+\mu_{d}}+\frac{\theta_{3} \lambda_{v a 2} \mu_{d}^{3}}{N_{v a 2} \beta_{a a} \theta_{3}+N_{v b 2} \beta_{b a} \theta_{4}+\mu_{d}}\right.$ $+\theta_{3} \kappa_{a 1} \kappa_{a 2} \mu_{v_{a}}+\theta_{3} \kappa_{a 1} \mu_{d} \mu_{v_{a}}+\theta_{3} \kappa_{a 2} \mu_{d} \mu_{v_{a}}+\theta_{3} \mu_{d}^{2} \mu_{v_{a}}+\theta_{3} \kappa_{a 1} \kappa_{a 2} \mu_{v_{b}} \theta_{2} \kappa_{a 1} \mu_{d} \mu_{v b}+\theta_{3} \kappa_{a 2} \mu_{d} \mu_{v b}+\theta_{3} \mu_{d}^{2} \mu_{v_{b}}$

$-\frac{\kappa_{a 2} \lambda_{v a 2} \mu_{d} \mu_{v_{b}}}{N_{v a 2} \beta_{a a} \theta_{3}+N_{v b 2} \beta_{b a} \theta_{4}+\mu_{d}}+\frac{\theta_{3} \kappa_{a 2} \lambda_{v a 2} \mu_{d} \mu_{v_{b}}}{N_{v a 2} \beta_{a a} \theta_{3}+N_{v b 2} \beta_{b a} \theta_{4}+\mu_{d}}-\frac{2 \lambda_{v a 2} \mu_{d}^{2} \mu_{v_{b}}}{N_{v a 2} \beta_{a a} \theta_{3}+N_{v b 2} \beta_{b a} \theta_{4}+\mu_{d}}+\frac{2 \theta_{3} \lambda_{v a 2} \mu_{d}^{2} \mu_{v_{b}}}{N_{v a 2} \beta_{a a} \theta_{3}+N_{v b 2} \beta_{b a} \theta_{4}+\mu_{d}}$ $+\theta_{3} \kappa_{a 1} \mu_{v_{a}} \mu_{v_{b}}+\theta_{3} \kappa_{a 2} \mu_{v_{a}} \mu_{v_{b}}+2 \theta_{3} \mu_{d} \mu_{v_{a}} \mu_{v_{b}}+\frac{1}{\left(N_{v a 2} \beta_{a a} \theta_{3}+N_{v b 2} \beta_{b a} \theta_{4}+\mu_{d}\right)^{2}}$

$N_{v b 2} \beta_{b a} \mu_{d}\left(N_{v a 2} \beta_{a a} \theta_{3}\left(-\theta_{3} \lambda_{v b 2}\left(\kappa_{a 1}+\mu_{d}+\mu_{v_{a}}\right)+\theta_{4}\left(\left(-1+\theta_{3}\right) \kappa_{a 2} \lambda_{v a 2}-\lambda_{v a 2}\left(\mu_{d}+\mu_{v_{b}}\right)\right.\right.\right.$

$\left.\left.+\theta_{3}\left(\lambda_{v b 2}\left(\kappa_{a 1}+\mu_{d}+\mu_{v_{a}}\right)+\lambda_{v a 2}\left(\mu_{d}+\mu_{v_{b}}\right)\right)\right)\right)+N_{v b 2} \beta_{b a} \theta_{4}\left(-\theta_{3} \lambda_{v b 2}\left(\kappa_{a 1}+\mu_{d}+\mu_{v_{a}}\right)+\theta_{4}\left(\left(-1+\theta_{3}\right) \kappa_{a 2} \lambda_{v a 2}\right.\right.$

$\left.\left.-\lambda_{v a 2}\left(\mu_{d}+\mu_{v_{b}}\right)+\theta_{3}\left(\lambda_{v b 2}\left(\kappa_{a 1}+\mu_{d}+\mu_{v_{a}}\right)+\lambda_{v a 2}\left(\mu_{d}+\mu_{v_{b}}\right)\right)\right)\right)+\mu_{d}\left(-\lambda_{v b 2}\left(-\lambda_{v a 2}+\theta_{3}\left(\kappa_{a 1}+\lambda_{v a 2}\right.\right.\right.$

$\left.\left.\left.\left.\left.+\mu_{d}+\mu_{v_{a}}\right)\right)+\theta_{4}\left(\left(-1+\theta_{3}\right) \kappa_{a 2} \lambda_{v a 2}-\lambda_{v a 2}\left(\lambda_{v b 2}+\mu_{d}+\mu_{v_{b}}\right)+\theta_{3}\left(\kappa_{a 1} \lambda_{v b 2}+\lambda_{v b 2}\left(\mu_{d}+\mu_{v_{a}}\right)+\lambda_{v a 2}\left(\lambda_{v b 2}+\mu_{d}+\mu_{v_{b}}\right)\right)\right)\right)\right)\right)$ 


$$
\begin{aligned}
& G_{5}=\frac{1}{\left(N_{v a 2} \beta_{a a} \theta_{3}+N_{v b 2} \beta_{b a} \theta_{4}+\mu_{d}\right)^{2}}\left(N_{v b 2}^{3} \beta_{a a}^{3} \theta_{3}^{3}\left(\kappa_{a 1}+\mu_{d}\right)\left(\kappa_{a 2}+\mu_{d}\right)\right. \\
& \mu_{v_{a}} \mu_{v_{b}}+\left(N_{v b 2} \beta_{b a} \theta_{4}+\mu_{d}\right)\left(\kappa_{a 1}+\mu_{d}\right) \mu_{v_{a}}\left(N_{v b 2} \beta_{b a}\left(-1+\theta_{4}\right) \lambda_{v b 2} \mu_{d}^{2}+\left(N_{v b 2} \beta_{b a} \theta_{4}+\mu_{d}\right)^{2}\right. \\
& \left.\left(\kappa_{a 2}+\mu_{d}\right) \mu_{v_{b}}\right)+N_{v a 2}^{2} \beta_{a a}^{2} \theta_{3}\left(N_{v b 2} \beta_{b a} \theta_{3}\left(-1+\theta_{4}\right) \lambda_{v b 2} \mu_{d}\left(\kappa_{a 1}+\mu_{d}\right) \mu_{v_{a}}+\left(N_{v b 2} \beta_{b a} \theta_{4}+\mu_{d}\right)\right. \\
& \left.\left(\kappa_{a 2}+\mu_{d}\right)\left(\left(-1+\theta_{3}\right) \lambda_{v a 2} \mu_{d}+3 \theta_{3}\left(\kappa_{a 1}+\mu_{d}\right) \mu_{v_{a}}\right) \mu_{v_{b}}\right)+N_{v a 2} \beta_{a a}\left(\mu _ { d } ^ { 2 } ( \kappa _ { a 2 } + \mu _ { d } ) \left(\left(-1+\theta_{3}\right) \lambda_{v a 2} \mu_{d}\right.\right. \\
& \left.\left.+3 \theta_{3}\left(\kappa_{a 1}+\mu_{d}\right) \mu_{v_{a}}\right) \mu_{v_{b}}\right)+N_{v b 2}^{2} \beta_{b a}^{2} \theta_{4}\left(\theta_{3}\left(-1+\theta_{4}\right) \lambda_{v b 2} \mu_{d}\left(\kappa_{a 1}+\mu_{d}\right) \mu_{v_{a}}+\theta_{4}\left(\kappa_{a 2}+\mu_{d}\right)\right. \\
& \left.\left(\left(-1+\theta_{3}\right) \lambda_{v a 2} \mu_{d}+3 \theta_{3}\left(\kappa_{a 1}+\mu_{d}\right) \mu_{v_{a}}\right) \mu_{v_{b}}\right)+N_{v b 2} \beta_{b a} \mu_{d}\left(\left(-1+\theta_{4}\right) \lambda_{v b 2} \mu_{d}+2 \theta_{3}\left(\kappa_{a 1}+\mu_{d}\right) \mu_{v_{a}}\right) \\
& \left.\left.\left.+2 \theta_{4}\left(\kappa_{a 2}+\mu_{d}\right)\left(\left(-1+\theta_{3}\right) \lambda_{v a 2} \mu_{d}+3 \theta_{3}\left(\kappa_{a 1}+\mu_{d}\right) \mu_{v_{a}}\right) \mu_{v_{b}}\right)\right)\right)
\end{aligned}
$$

Where:

$$
\begin{gathered}
\left(N_{t c} N_{v a 1} \beta_{a c} \lambda_{v a 1} \lambda_{v b 1} \mu_{d}-\lambda_{v b 1}\left(N_{v b 1} \beta_{b c}+\mu_{d}\right)\right. \\
\theta_{1}=\frac{\left.\left(\kappa_{c 1}+\mu_{d}\right) \mu_{v_{a}}+N_{v a 1} \beta_{a c} \lambda_{v a 1}\left(\kappa_{c 2}+\mu_{d}\right) \mu_{v_{b}}\right)}{\left(N _ { v a 1 } \beta _ { a c } \left(N_{t c} \lambda_{v a 1} \lambda_{v b 1} \mu_{d}+\lambda_{v b 1}\right.\right.} \\
\left.\left.\left(\kappa_{c 1}+\mu_{d}\right) \mu_{v_{a}}+\lambda_{v a 1}\left(\kappa_{c 2}+\mu_{d}\right) \mu_{v_{b}}\right)\right)
\end{gathered}
$$

$\theta_{2}=\frac{N_{t c} N_{v b 1} \beta_{b c} \lambda_{v b 1} \mu_{d}-\left(N_{v a 1} \beta_{a c} \theta_{1}+\mu_{d}\right)\left(\kappa_{c 2}+\mu_{d}\right) \mu_{v_{b}}}{N_{v b 1} \beta_{b c}\left(N_{t c} \lambda_{v b 1} \mu_{d}+\left(\kappa_{c 2}+\mu_{d}\right) \mu_{v_{b}}\right)}$

$$
\begin{aligned}
& \left(N_{t a} N_{v a 2} \beta_{a a} \lambda_{v a 2} \lambda_{v b 2} \mu_{d}-\lambda_{v b 2}\left(N_{v b 2} \beta_{b a}+\mu_{d}\right)\right. \\
\theta_{3}= & \frac{\left.\left(\kappa_{a 1}+\mu_{d}\right) \mu_{v_{a}}+N_{v a 2} \beta_{a a} \lambda_{v a 2}\left(\kappa_{a 2}+\mu_{d}\right) \mu_{v_{b}}\right)}{\left(N _ { v a 2 } \beta _ { a a } \left(N_{t a} \lambda_{v a 2} \lambda_{v b 2} \mu_{d}\right.\right.} \\
& \left.\left.+\lambda_{v b 2}\left(\kappa_{a 1}+\mu_{d}\right) \mu_{v_{a}}+\lambda_{v a 2}\left(\kappa_{a 2}+\mu_{d}\right) \mu_{v_{b}}\right)\right)
\end{aligned}
$$$$
\theta_{4}=\frac{N_{t a} N_{v b 2} \beta_{b a} \lambda_{v b 2} \mu_{d}-\left(N_{v a 2} \beta_{a a} \theta_{3}+\mu_{d}\right)\left(\kappa_{a 2}+\mu_{d}\right) \mu_{v_{b}}}{N_{v b 2} \beta_{b a}\left(N_{t a} \lambda_{v b 2} \mu_{d}+\left(\kappa_{a 2}+\mu_{d}\right) \mu_{v_{b}}\right)}
$$

From the characteristic Equation (11a)-(11b), the eigenvalues are found by solving $\left(\eta^{5}+D_{1} \eta^{4}+D_{2} \eta^{3}+D_{3} \eta^{2}+D_{4} \eta+D_{5}\right)=0$ in children and in adult $\left(\eta^{5}+G_{1} \eta^{4}+G_{2} \eta^{3}+G_{3} \eta^{2}+G_{4} \eta+G_{5}\right)=0$, when $\mathrm{T}_{1}=\mathrm{D}_{1}$

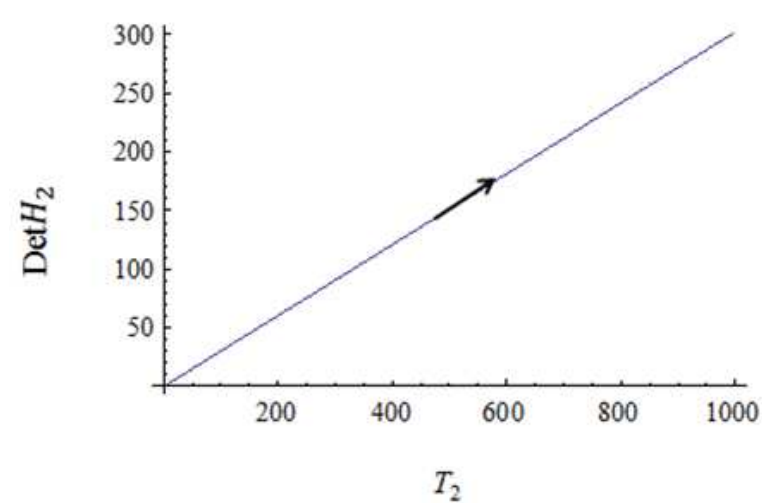

and $\mathrm{G}_{1}$ for children and adult, $T_{2}=D_{2}$ and $G_{2}$, for children and adult, $T_{3}=D_{3}$ and $G_{3}$ for children and adult $T_{4}=D_{4}$ and $G_{4}$ for children and adult, $T_{5}=D_{5}$ and $\mathrm{G}_{5}$ for children and adult. The five eigenvalues have negative real parts if they satisfy RouthHurwitz criteria (14b)-(14e) (Edelstein-Keshet, 1988), each equilibrium state is locally asymptotically stable, when it satisfies the following conditions:

$\operatorname{det} H_{1}=T_{1}>0$

$\operatorname{det} H_{2}=T_{1} T_{2}-T_{3}>0$

$\operatorname{det} H_{3}=T_{1} T_{2} T_{3}-T_{3}^{2}-T_{1}^{2} T_{4}>0$

$\operatorname{det} H_{4}=T_{1} T_{2} T_{3} T_{4}-T_{3}^{2} T_{4}-T_{1}^{2} T_{4}^{2}>0$

$\operatorname{det} H_{5}=T_{1} T_{2} T_{3} T_{4} T_{5} T_{3}^{2} T_{4} T_{5} T_{1}^{2} T_{4}^{2} T_{5} T_{1} T_{2}^{2} T_{5}^{2}$

$+T_{2} T_{3} T_{5}^{2}+2 T_{1} T_{4} T_{5}^{2} T_{5}^{3}>0$

We check the stability of endemic equilibrium state by using the Routh-Hurwitz conditions (14a)(14e), the results are given in Fig. 3.

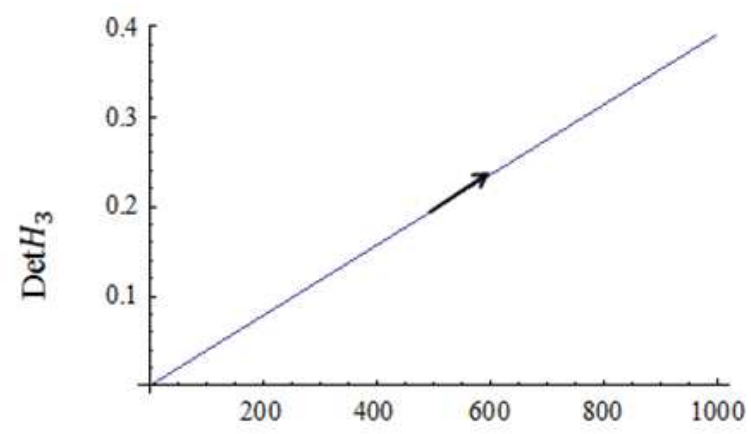

$T_{2}$ 


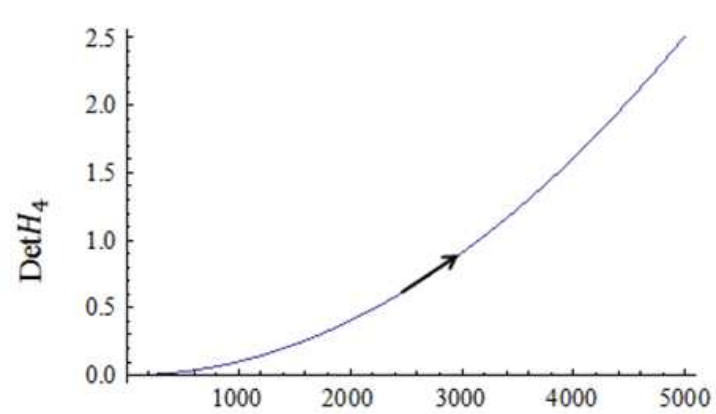

$T_{2}$

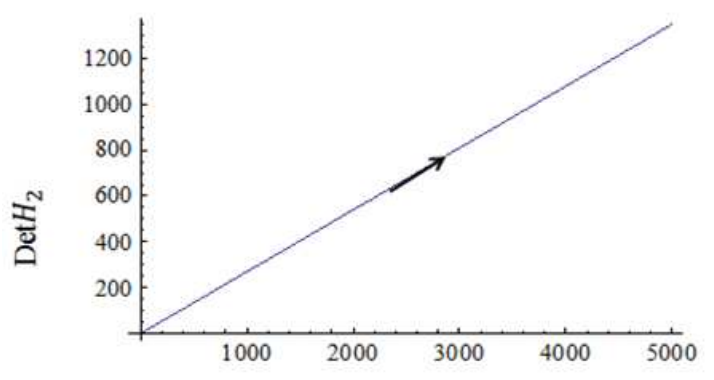

$T_{2}$

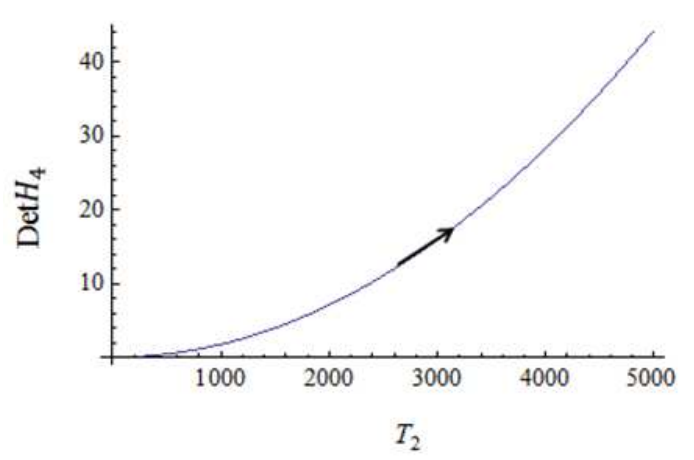

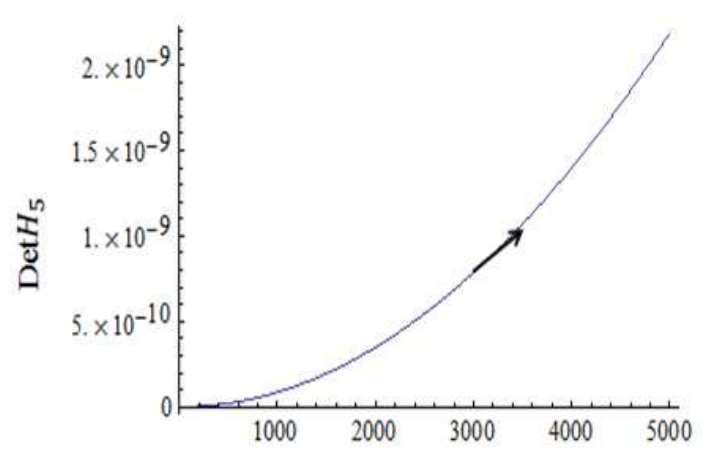

$T_{2}$

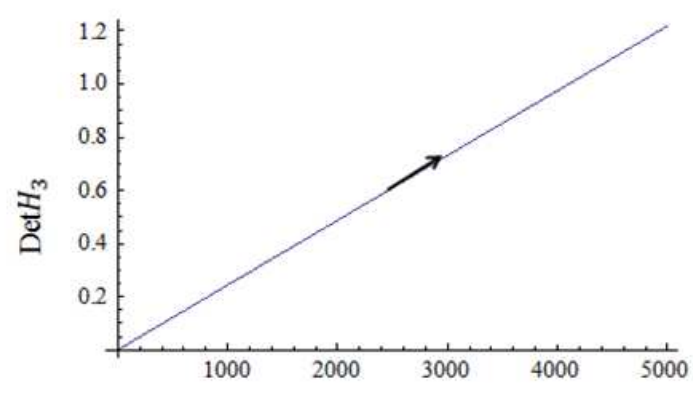

$T_{2}$

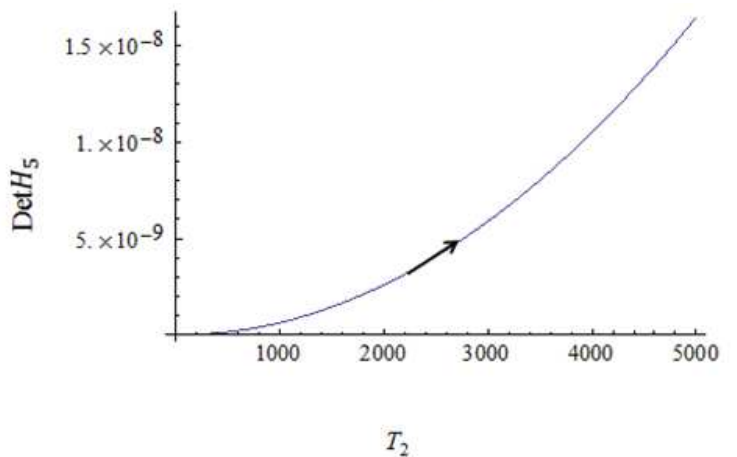

$T_{2}$

(b)

Fig. 3. The parameter spaces for endemic disease equilibrium state, which satisfies the Routh-Hurwitz conditions, plotted onto $\left(\kappa_{c 1}, \operatorname{det} H_{2}\right),\left(\kappa_{c 1}, \operatorname{det} H_{3}\right),\left(\kappa_{c 1}, \operatorname{det} H_{4}\right),\left(\kappa_{c 1}, \operatorname{det} H_{5}\right),\left(\kappa_{a 1}, \operatorname{det} H_{2}\right),\left(\kappa_{a 1}, \operatorname{det} H_{3}\right),\left(\kappa_{a 1}, \operatorname{det} H_{4}\right)$ and $\left(\kappa_{a 1}, \operatorname{det} H_{5}\right)$, respectively. The values of parameter are follows: $(a)_{\kappa_{c 1}=1 /(17 / 2), \kappa_{c 2}=1 /(19 / 2), \quad \mu_{d}=1 /\left(365^{*} 74.6\right)}$ day $^{-1}$, $N_{t c}=6000, \quad N_{v a 1}=5000, \quad N_{v b 1}=2500, \quad \beta_{a c}=0.2, \quad \beta_{b c}=0.0714, \quad \lambda_{v a 1}=0.00000000576, \quad \lambda_{v b 1}=0.00000435, \quad \alpha_{a}=0.08$, $\alpha_{b}=0.047$ and $N_{t}=100,000, \quad(\mathrm{~b})_{\kappa_{a 1}=1 /(19 / 2)}, \kappa_{a 2}=1 /(21 / 2), \quad \mu_{d}=1 /(365 * 74.6) \mathrm{day}^{-1}, \quad N_{t a}=4000, \quad N_{v a 2}=7000, \quad N_{v b 2}=4300$, $\beta_{a a}=0.1667, \beta_{b a}=0.125, \lambda_{v a 2}=0.00000000176, \lambda_{v b 2}=0.000000835, \alpha_{a}=0.07, \alpha_{b}=0.027$ and $N_{t}=100000$. From the above figures, the Routh-Hurwitz conditions are satisfies for $S_{0}>1$

\section{Numerical Results}

We consider the numerical solutions for dengue virus transmission. The main effect of introducing an age structure into the model is to change the definition of the basic reproductive rate. The parameters in this study are determined by the real life observations. The values of the parameters are as follows: $\mu_{d}=1 /\left(365^{*} 74.6\right)$ day $^{-1}$, corresponding to life expectancy of 74.6 years for human; $\kappa_{c 1}=1 /(8.5)$ and $\kappa_{c 2}=1 /(9.5)$ corresponding to the 8.5 days and 9.5 days of recovering due to biting of Aedes aegypti and Aedes albopictus, respectively. The death rate of mosquitoes are 1/28 per day and 
$1 / 35$ per day satisfies to the life time of 28 days for Aedes aegypti and the life time of 35 days for Aedes albopictus, respectively $\kappa_{\mathrm{a} 1}=1 /(9.5)$ and $\kappa_{\mathrm{a} 1}=$ $1 /(10.5)$ corresponding to the 9.5 days and 10.5 days of recovering of adult human population due to biting of Aedes aegypti and Aedes albopictus, respectively. The other parameters are arbitrary chosen. Numerical solutions of (4a)-(4j) are shown in Fig. 4-9.
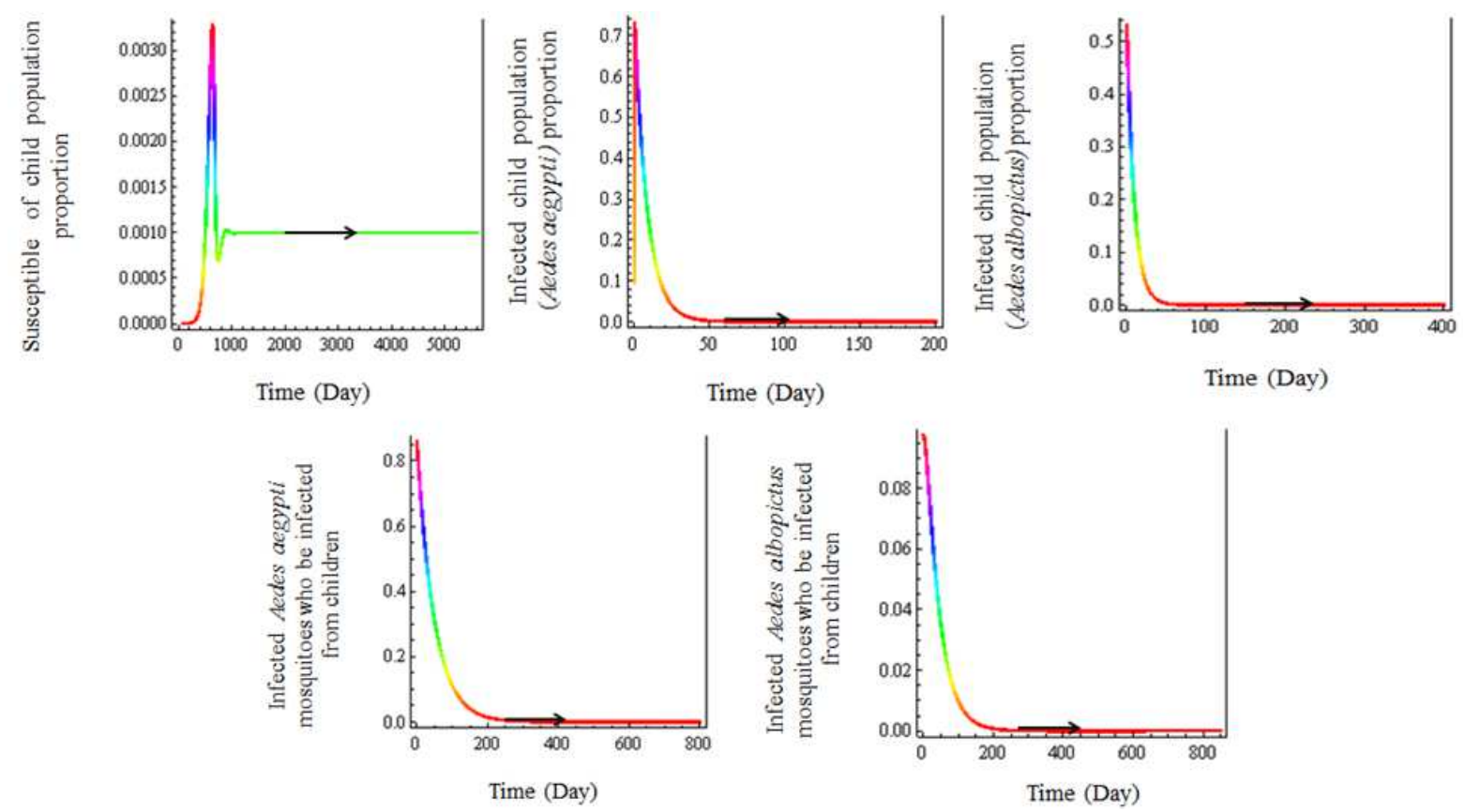

Fig. 4. Time series solutions of $S_{c}, I_{c 1}, I_{c 2}, I_{v a 1}$ and $I_{v b 1}$ respectively. For $S_{0}<1$ and $\mathrm{S}_{0 \mathrm{c}}=0.000023944$ with parameters are following: $\mu_{v a 1}=1 / 49, \quad \mu_{v b 1}=1 / 39, \quad N_{t c}=71000, \quad N_{v a 1}=5800, \quad N_{v b 1}=10000, \quad \beta_{a c}=0.0239, \quad \beta_{b c}=0.0333, \quad \lambda_{v a 1}=0.00000000000347$, $\lambda_{v b 1}=0.0000000675, \alpha_{a}=0.07, \alpha_{b}=0.027$ and $N_{t}=100,000$. The proportions of populations $\left(S_{c}^{\prime}, I_{c 1}^{\prime}, I_{c 2}^{\prime}, I_{v a 1}^{\prime}, I_{v b 1}^{\prime}\right)$ approach to the disease free equilibrium state $(1,0,0,0,0)$
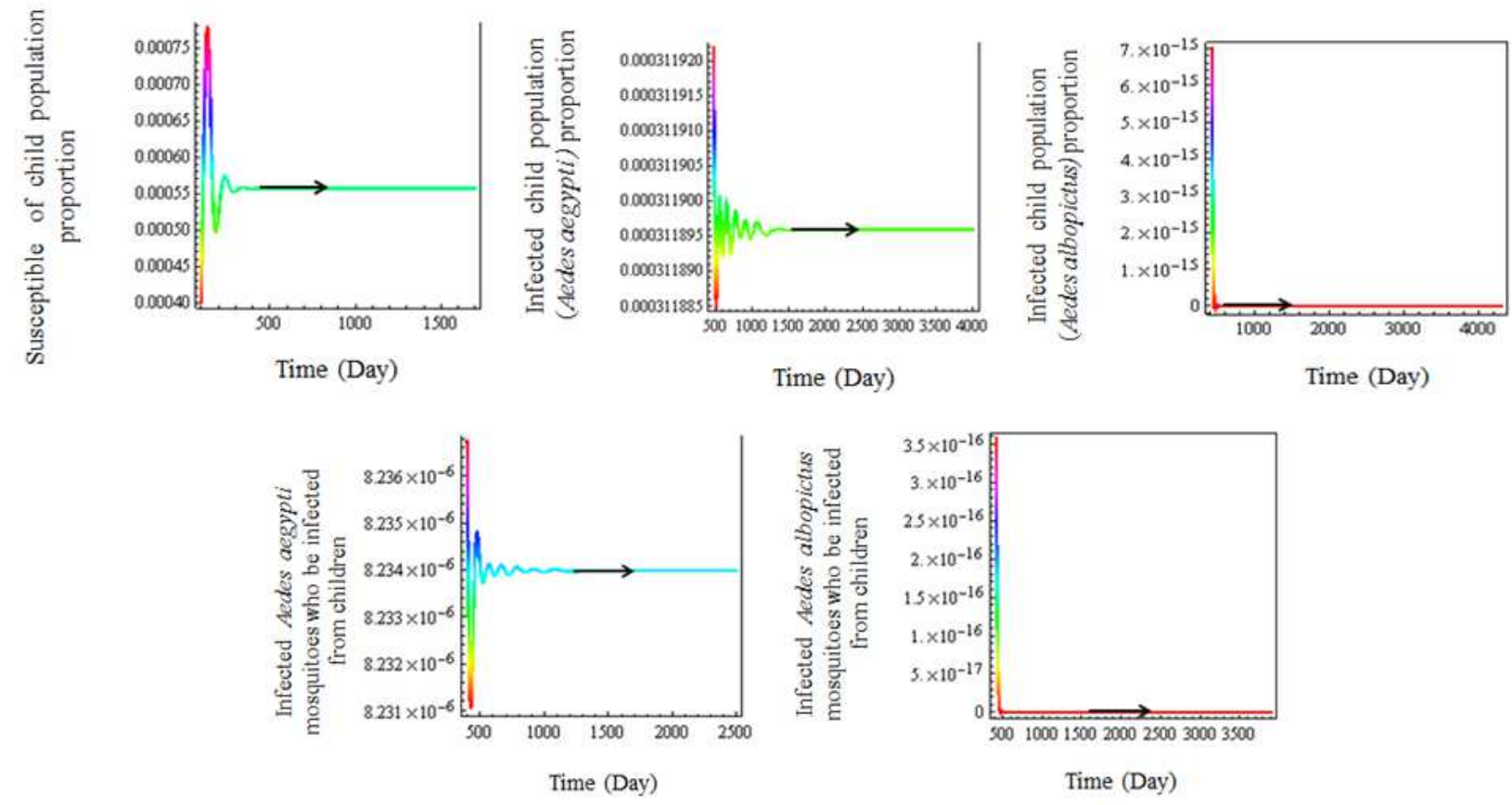

(a) 


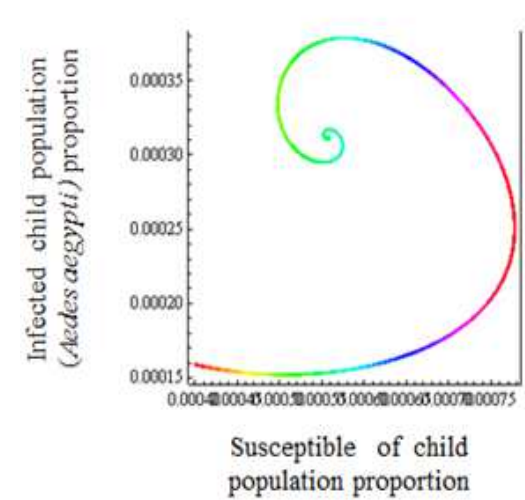

population proportion

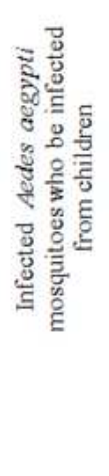

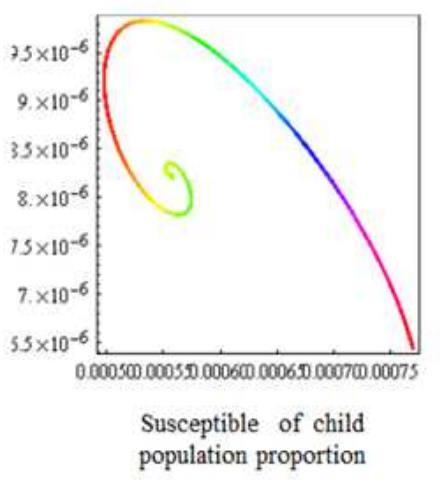

(b)

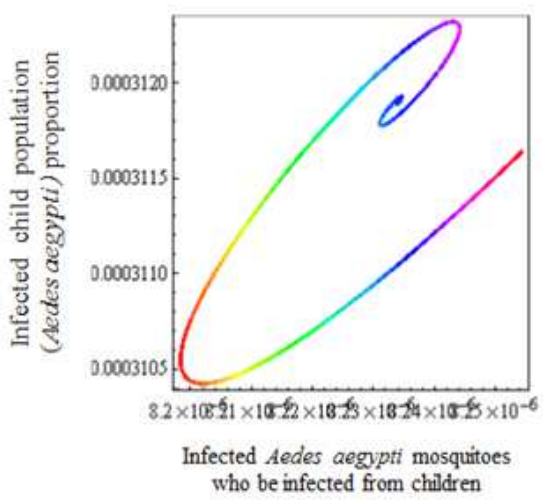

Fig. 5. (a) Time series solutions of $S_{c}, I_{c 1}, I_{c 2}, I_{v a 1}, I_{v b 1}$. Values of parameters in the model are following: $\mu_{v a 1}=1 / 7$, $\mu_{v b 1}=1 / 14, \quad N_{t c}=50000, \quad N_{v a 1}=32000, \quad N_{v b 1}=17000, \quad \beta_{a c}=0.2, \quad \beta_{b c}=0.125, \quad \lambda_{v a 1}=0.0000000058, \quad \lambda_{v b 1}=0.00000000465$, $\alpha_{a}=0.026, \alpha_{b}=0.009$ and $N_{t}=100,000$, when $\mathrm{S}_{0 \mathrm{c}}=174.473$. (b) Numerical solutions projected onto $\left(S_{c}^{\prime}, I_{c 1}^{\prime}\right), \quad\left(S_{c}^{\prime}, I_{v a 1}^{\prime}\right),\left(I_{v a 1}^{\prime}, I_{c 1}^{\prime}\right)$. The solutions oscillate to the endemic equilibrium state $\left(S_{c}^{*}, I_{c 1}^{*}, I_{c 2}^{*}, I_{v a 1}^{*}, I_{v b 1}^{*}\right) \quad$ where $\quad S_{c}^{*}=0.000556913, I_{c 1}^{*}=0.0003, \quad I_{c 2}^{*}=1.6622 \times 10^{-14}, \quad I_{v a 1}^{*}=8.23484 \times 10^{-6} \quad$ and $I_{v b 1}^{*}=7.38289 \times 10^{-17}$, respectively
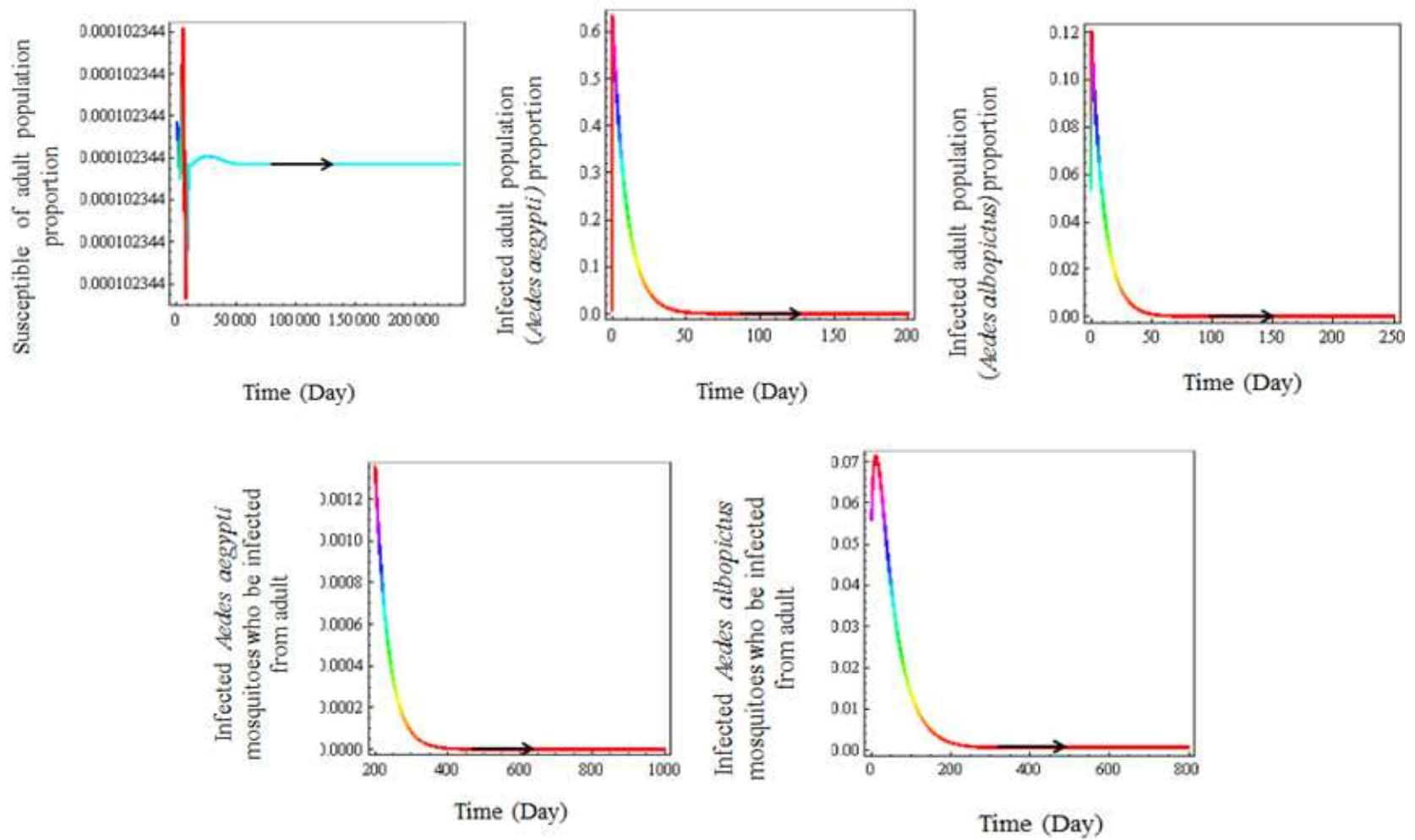

Fig. 6. Time series solution of $S_{a}, I_{a 1}, I_{a 2}, I_{v a 2}$ and $I_{v b 2}$ respectively. For $S_{0}<1$ and $\mathrm{S}_{0 \mathrm{a}}=0.0307919$ with parameters are following: $\quad \mu_{v a 2}=1 / 36, \quad \mu_{v b 2}=1 / 46, \quad N_{t a}=61000, \quad N_{v a 2}=4800, \quad N_{v b 2}=10000, \quad \beta_{a a}=0.03225, \quad \beta_{b a}=0.02941$, $\lambda_{v a 2}=0.000000000076, \quad \lambda_{v b 2}=0.000000000664, \quad \alpha_{a}=0.04, \quad \alpha_{b}=0.06$ and $N_{t}=100,000 . \quad$ The proportions of populations $\left(S_{a}^{\prime}, I_{a 1}^{\prime}, I_{a 2}^{\prime}, I_{v a 2}^{\prime}, I_{v b 2}^{\prime}\right)$ approach to the disease free equilibrium state $(1,0,0,0,0)$ 

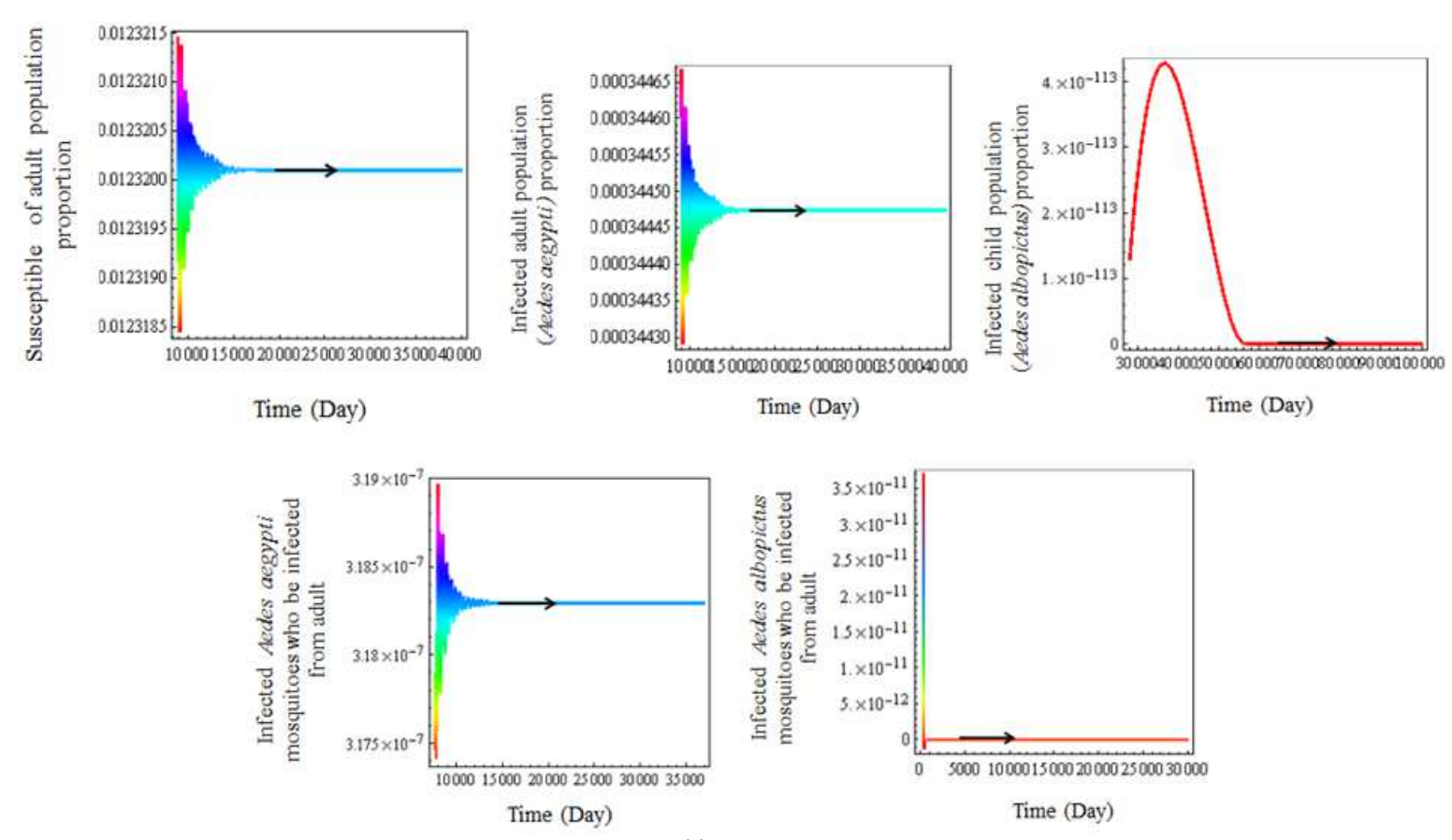

(a)
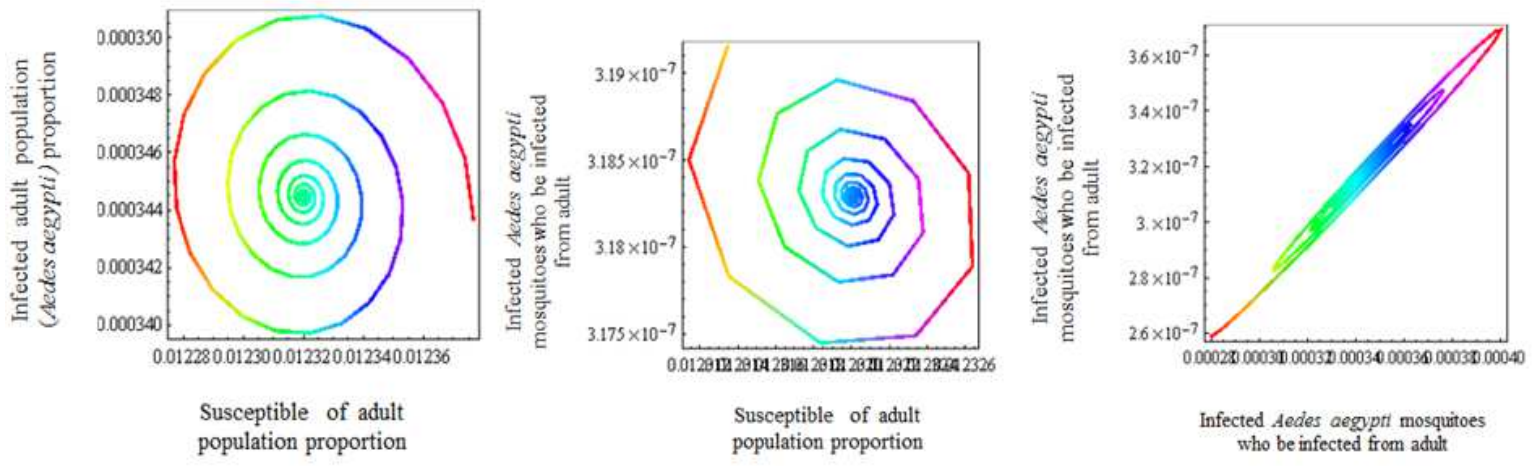

(b)

Fig. 7. (a) Time series solutions of $S_{a}, I_{a 1}, I_{a 2}, I_{v a 2}, I_{v b 2}$. Values of parameters in the model are following: $\mu_{v a 2}=1 / 7$, $\mu_{v b 2}=1 / 13, \quad N_{t a}=30000, \quad N_{v a 2}=37000, \quad N_{v b 2}=19000, \quad \beta_{a a}=0.25, \quad \beta_{b a}=0.1428, \quad \lambda_{v a 2}=0.0000000044$, $\lambda_{v b 2}=0.000000000335, \alpha_{a}=0.02, \alpha_{b}=0.07$ and $N_{t}=100,000$, where $\mathrm{S}_{0 \mathrm{a}}=21.7785$ in adult. (b) Numerical solutions projected onto $\left(S_{a}^{\prime}, I_{a 1}^{\prime}\right), \quad\left(S_{a}^{\prime}, I_{v a 2}^{\prime}\right), \quad\left(I_{v a 2}^{\prime}, I_{a 1}^{\prime}\right)$. The solutions oscillate to the endemic equilibrium state $\left(S_{a}^{*}, I_{a 1}^{*}, I_{a 2}^{*}, I_{v a 2}^{*}, I_{v b 2}^{*}\right)$ where $S_{c}^{*}=0.0123201, \quad I_{c 1}^{*}=0.000344744, I_{c 2}^{*}=6.9961 \times 10^{-14}, \quad I_{v a 1}^{*}=3.18294 \times 10^{-7} \quad$ and $\quad I_{v b 1}^{*}=1.40069 \times 10^{-16}$, respectively

Case A.1, in children, we consider the locally asymptotically stable of disease free equilibrium state, when $\varepsilon=0$ as shown in Fig. 4.

Case A.2, in children, we consider the locally asymptotically stable of endemic equilibrium state, when $\varepsilon=0$ as shown in Fig. 5.

Case B.1, in adult, we consider the locally asymptotically stable of disease free equilibrium state, when $\varepsilon=0$ as shown in Fig. 6.

Case B.2, in adult, we consider the locally asymptotically stable of endemic equilibrium state, when $\varepsilon=0$ as shown in Fig. 7.

Case $C$, in children, when $\varepsilon \neq 0$ as shown in Fig. 8.

Case $\mathrm{D}$, in adult, when $\varepsilon \neq 0$ as shown in Fig. 9. 

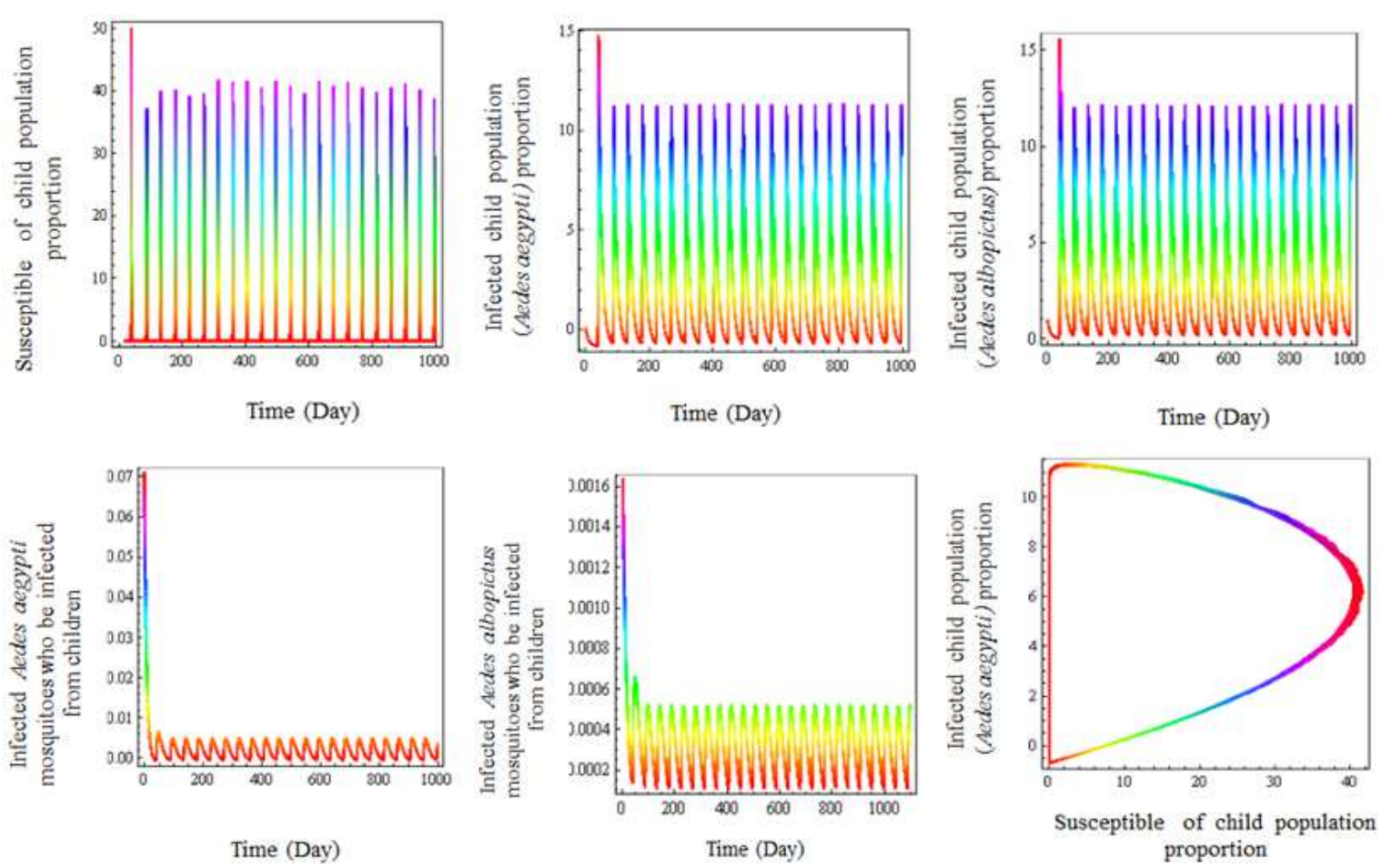

(a)
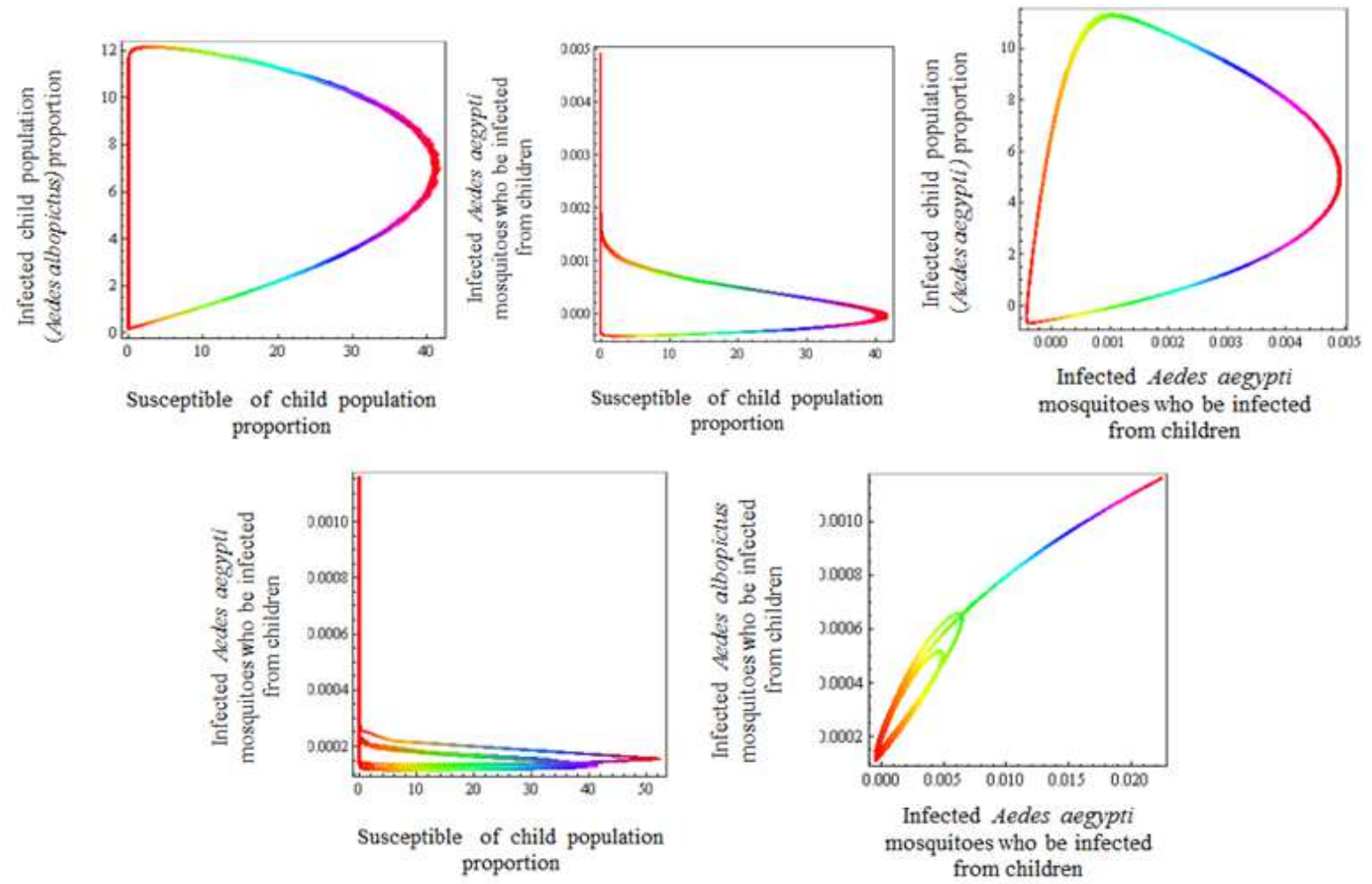

(b)

Fig. 8. (a) Time series solutions of $S_{c}, I_{c 1}, I_{c 2}, I_{v a 1}, I_{v b 1}$. Values of parameters in the model are following: $N_{t c}=50000, N_{v a 1}=32000$, $N_{v b 1}=17000, \beta_{a c}=0.2, \beta_{b c}=0.125, \lambda_{v a 1}=0.0000000028, \lambda_{v b 1}=0.000000000165, \alpha_{a}=0.005, \alpha_{b}=0.004$ and $N_{t}=100,000$ and $\mathrm{S}_{0 c}$ $=22.8627$, (b) Numerical solutions projected onto $\left(S_{c}^{\prime}, I_{c 1}^{\prime}\right),\left(S_{c}^{\prime}, I_{c 2}^{\prime}\right),\left(S_{c}^{\prime}, I_{v a 1}^{\prime}\right),\left(S_{c}^{\prime}, I_{v b 1}^{\prime}\right),\left(I_{v a 1}^{\prime}, I_{c 1}^{\prime}\right),\left(I_{v a 1}^{\prime}, I_{v b 1}^{\prime}\right)$. Limit1 cycles are produced in this case 

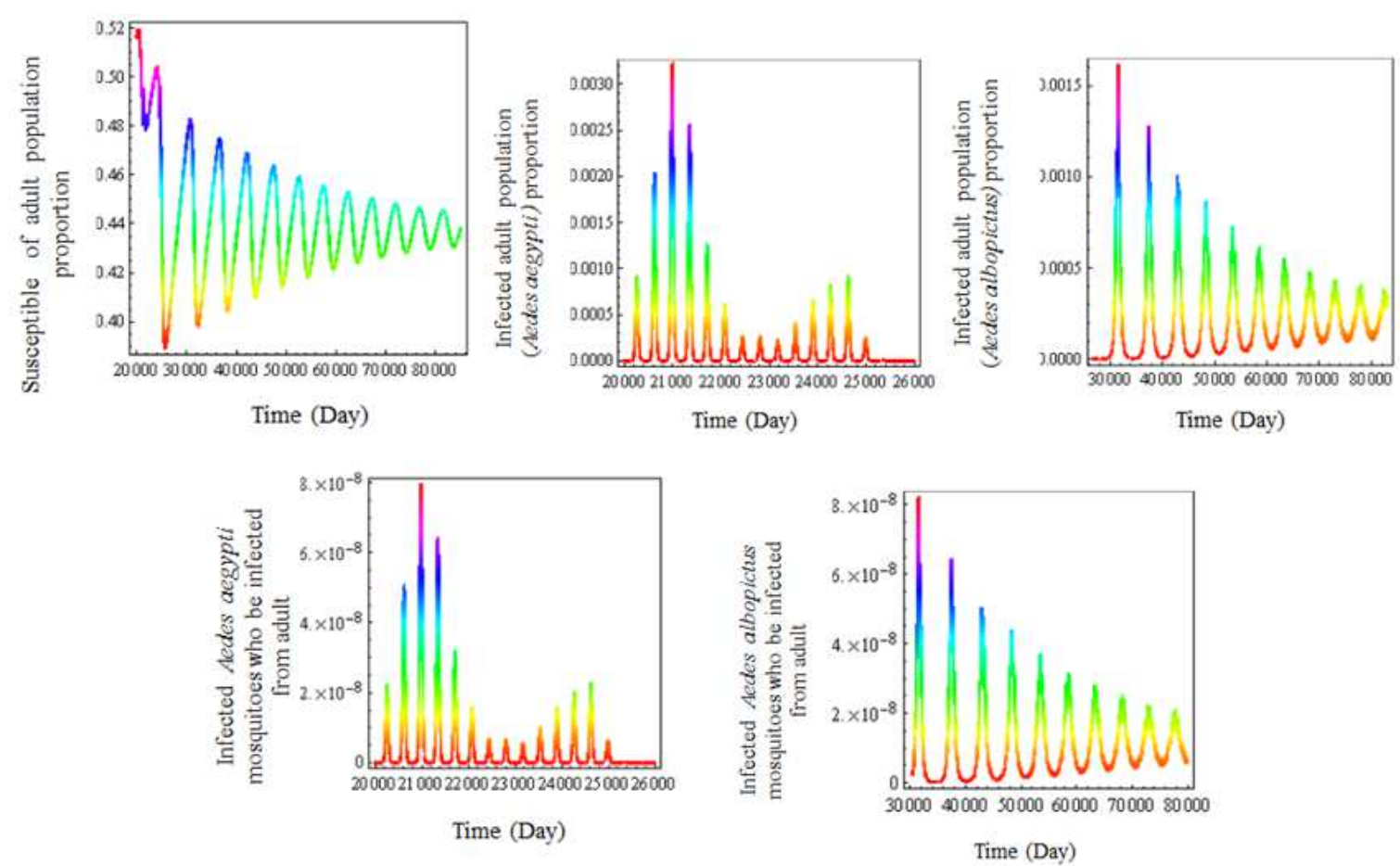

(a)
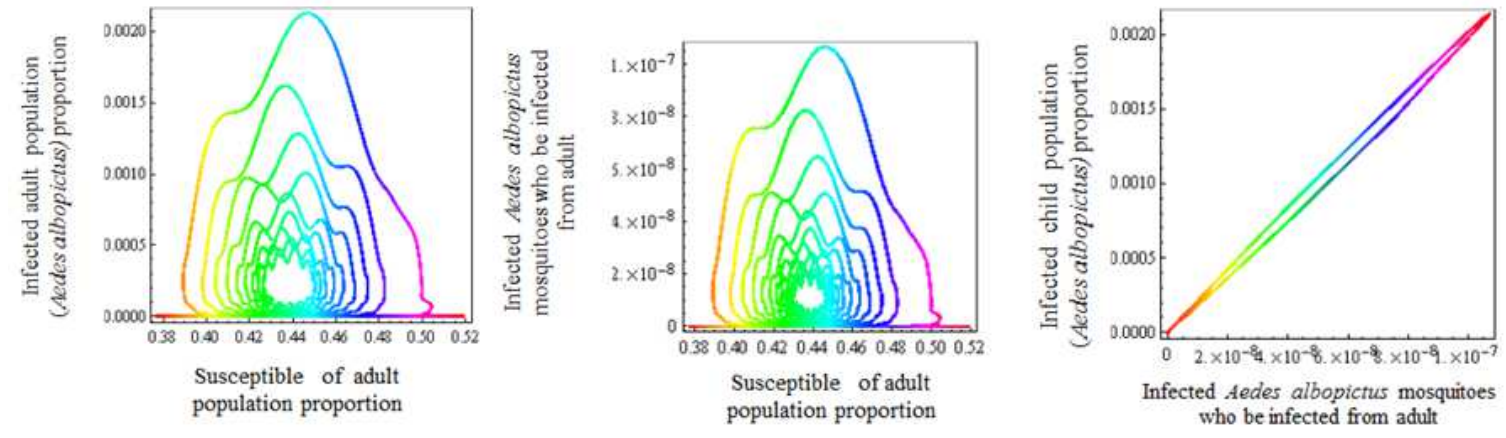

(b)

Fig. 9. (a) Time series solutions of $S_{a}, I_{a 1}, I_{a 2}, I_{v a 2}, I_{v b 2}$. Values of parameters in the model are following: $N_{t a}=50000$, $N_{v a 2}=34000, N_{v b 2}=30000, \beta_{a a}=0.25, \beta_{b a}=0.1428, \lambda_{v a 2}=0.0000000075, \lambda_{v b 2}=0.000000000625, \alpha_{a}=0.02, \alpha_{b}=0.07$ and $N_{t}=100,000$, when $\mathrm{S}_{0 \mathrm{a}}=9.26764$. (b) Numerical solutions projected onto $\left(S_{a}^{\prime}, I_{a 2}^{\prime}\right),\left(S_{a}^{\prime}, I_{v b 2}^{\prime}\right),\left(I_{v b 2}^{\prime}, I_{a 2}^{\prime}\right)$. The solutions oscillate to the endemic equilibrium state $\left(S_{a}^{*}, I_{a 1}^{*}, I_{a 2}^{*}, I_{v a 2}^{*}, I_{v b 2}^{*}\right)$ are limit cycles

\section{Discussion}

Several investigations have been conducted using the SIR and SI models. The SIR and SI models which provide suitable for the states of children and adult in two species are used in this study. (Aedes aegypti and Aedes albopictus).

The basic reproductive number of equations $(4 \mathrm{a})-(4 \mathrm{j})$ is defined as follows (Chong et al., 2013):

$$
\begin{aligned}
& 2 N_{v b 1} \alpha_{b} \beta_{b c} \lambda_{v b 1}\left(\kappa_{c 1}+\mu_{d}\right) \mu_{v_{a}} \rho_{v b} \\
& +N_{v a 1} \beta_{a c} \lambda_{v a 1}\left(2\left(N_{t c} \lambda_{v b 1} \mu_{d}+\left(\kappa_{c 2}+\mu_{d}\right) \mu_{v_{b}}\right)\right.
\end{aligned}
$$

$$
\begin{gathered}
S_{0}=\max \left\{\frac{\left.\left(2+\alpha_{a} \rho_{v a}\right)+2 N_{t c} \lambda_{v b 1} \mu_{d}\left(\alpha_{a}+\rho_{v a}\right)\right)}{\left(2 \lambda _ { v b 1 } ( \kappa _ { c 1 } + \mu _ { d } ) \mu _ { v _ { a } } \left(2 \mu_{d}+N_{v b 1} \beta_{b c}\right.\right.},\right. \\
\left.\left(2+\alpha_{b} \rho_{v b}\right)\right)+2 N_{v a 1} \beta_{a c} \lambda_{v a 1}\left(\alpha_{a}\left(\kappa_{c 2}+\mu_{d}\right)\right. \\
\left.\left.\mu_{v_{b}} \rho_{v a}+N_{t c} \lambda_{v b 1} \mu_{d}\left(\alpha_{a} \rho_{v a}+\left(\alpha_{a}+\rho_{v a}\right) \rho_{v b}\right)\right)\right) \\
2 N_{v b 2} \alpha_{b} \beta_{b a} \lambda_{v b 2}\left(\kappa_{a 1}+\mu_{d}\right) \mu_{v_{a}} \rho_{v b} \\
+N_{v a 2} \beta_{a a} \lambda_{v a 2}\left(2\left(N_{t a} \lambda_{v b 2} \mu_{d}+\left(\kappa_{a 2}+\mu_{d}\right) \mu_{v_{b}}\right)\right. \\
\left.\frac{\left.\left(2+\alpha_{a} \rho_{v a}\right)+2 N_{t a} \lambda_{v b 2} \mu_{d}\left(\alpha_{a}+\rho_{v a}\right)\right)}{\left(2 \lambda_{v b 2}\left(\kappa_{a 1}+\mu_{d}\right) \mu_{v a}\left(2 \mu_{d}+N_{v b 2} \beta_{b a}\left(2+\alpha_{b} \rho_{v b}\right)\right)\right.}\right\} \\
+2 N_{v a 2} \beta_{a a} \lambda_{v a 2}\left(\alpha_{a}\left(\kappa_{a 2}+\mu_{d}\right) \mu_{v b} \rho_{v a}\right. \\
\left.\left.+N_{t a} \lambda_{v b 2} \mu_{d}\left(\alpha_{a} \rho_{v a}+\left(\alpha_{a}+\rho_{v a}\right) \rho_{v b}\right)\right)\right)
\end{gathered}
$$


Table 3. Determination of the values $S_{0}$ of infected mosquitoes

\begin{tabular}{lllll}
\hline $\mathrm{I}_{\mathrm{va} 1}$ value & $\mathrm{I}_{\mathrm{vb} 1}$ value & $\mathrm{I}_{\mathrm{va} 2}$ value & $\mathrm{I}_{\mathrm{va} 2}$ value & $\mathrm{S}_{0}$ value \\
\hline $2.5687 \times 10-26$ & & $2.3860 \times 10-18$ & $6.44744 \times 10-7$ & 0.00310 \\
0 & 0.000065 & - & - & 0.00732 \\
- & 0.00036 & - & - & 0.06795 \\
- & - & $4.9155 \times 10-23$ & 0.0007175 & 0.68842 \\
$8.234 \times 10-6$ & - & $7.3376 \times 10-14$ & $1.07169 \times 10-8$ & 3.61291 \\
$3.16331 \times 10-5$ & $7.982 \times 10-17$ & - & - & 38.6066 \\
- & $1.118 \times 10-6$ & - & - & 80.3505 \\
\hline
\end{tabular}

$\mathrm{S}_{0}$ describes the number of infectious human produced from primary infection of children and adult. Using the initial values and parameter values from data, the obtained result of threshold parameter value $\mathrm{S}_{0}$ for $\mathrm{S}_{0 \mathrm{c}}$ and $\mathrm{S}_{0 \mathrm{a}}$ can be rewritten in mathematical form as follows:

$$
\begin{aligned}
& 2 N_{v b 1} \alpha_{b} \beta_{b c} \lambda_{v b 1}\left(\kappa_{c 1}+\mu_{d}\right) \mu_{v_{a}} \rho_{v b} \\
& +N_{v a 1} \beta_{a c} \lambda_{v a 1}\left(2 \left(N_{t c} \lambda_{v b 1} \mu_{d}+\left(\kappa_{c 2}+\mu_{d}\right)\right.\right. \\
S_{0 c}= & \frac{\left.\left.\mu_{v_{b}}\right)\left(2+\alpha_{a} \rho_{v a}\right)+2 N_{t c} \lambda_{v b 1} \mu_{d}\left(\alpha_{a}+\rho_{v a}\right)\right)}{\left(2 \lambda _ { v b 1 } ( \kappa _ { c 1 } + \mu _ { d } ) \mu _ { v _ { a } } \left(2 \mu_{d}+N_{v b 1} \beta_{b c}\right.\right.} \text { in children } \\
& \left.\left(2+\alpha_{b} \rho_{v b}\right)\right)+2 N_{v a 1} \beta_{a c} \lambda_{v a 1}\left(\alpha_{a}\left(\kappa_{c 2}+\mu_{d}\right)\right. \\
\left.\left.\mu_{v_{b}} \rho_{v a}+N_{t c} \lambda_{v b 1} \mu_{d}\left(\alpha_{a} \rho_{v a}+\left(\alpha_{a}+\rho_{v a}\right) \rho_{v b}\right)\right)\right) & \\
& 2 N_{v b 2} \alpha_{b} \beta_{b a} \lambda_{v b 2}\left(\kappa_{a 1}+\mu_{d}\right) \mu_{v_{a}} \rho_{v b}+ \\
& N_{v a 2} \beta_{a a} \lambda_{v a 2}\left(2\left(N_{t a} \lambda_{v b 2} \mu_{d}+\left(\kappa_{a 2}+\mu_{d}\right) \mu_{v_{b}}\right)\right. \\
S_{0 a}= & \frac{\left.\left(2+\alpha_{a} \rho_{v a}\right)+2 N_{t a} \lambda_{v b 2} \mu_{d}\left(\alpha_{a}+\rho_{v a}\right)\right)}{\left(2 \lambda _ { v b 2 } ( \kappa _ { a 1 } + \mu _ { d } ) \mu _ { v _ { a } } \left(2 \mu_{d}+N_{v b 2} \beta_{b a}\right.\right.} \\
& \left.\left(2+\alpha_{b} \rho_{v b}\right)\right)+2 N_{v a 2} \beta_{a a} \lambda_{v a 2}\left(\alpha_{a}\left(\kappa_{a 2}+\mu_{d}\right)\right. \\
& \left.\left.\mu_{v_{b}} \rho_{v a}+N_{t a} \lambda_{v b 2} \mu_{d}\left(\alpha_{a} \rho_{v a}+\left(\alpha_{a}+\rho_{v a}\right) \rho_{v b}\right)\right)\right)
\end{aligned}
$$

The reproductive rate is depend on the number of infected mosquitoes $\mathrm{I}_{\mathrm{va} 1}, \mathrm{I}_{\mathrm{vb} 1}$ in children and $\mathrm{I}_{\mathrm{va} 2}, \mathrm{I}_{\mathrm{vb} 2}$ in adult.

From the above table (Table 3), we will see that if the number of infected mosquitoes is increased, the basic reproductive rate is also increased.

Moreover, we consider the effect of sinusoidal variation $(\varepsilon)$, we will see that if $\varepsilon=0$, then the solutions oscillation to the steady state. The limit cycle occurs for $\varepsilon \neq 0$. Thus the limit cycles occurs while there is the seasonal variation of mosquitoes (Aedes aegypti and Aedes albopictus). It can be seen that the dynamical behavior of the endemic state change while there is the influence of season.

\section{Conclusion}

The basic reproductive number of disease is defined by $\tilde{S}_{0}=\sqrt{S_{0}}$. This value is the threshold condition for the existence of the endemic state. When $\mathrm{S}_{0} \leq 1$, the solutions oscillate to disease free equilibrium state, whereas $\mathrm{S}_{0}>$ 1 , the solutions oscillate to the endemic state. The behaviors of the proportion of susceptible, infective human into two classes (a child class and an adult class) and infective vectors of the two species (Aedes aegypti and Aedes albopictus) are initially positive. If this can be seen as follow; the infective human are introduced into the susceptible is bitten during each period, by the fraction $\frac{b N_{h}}{\left(N_{h}+m\right)}\left(\frac{1}{\mu_{v}}\right)$ (that the biting rate $b$ of mosquitoes is the average number of bites per mosquito per day, $\mu_{v}$ is the per capita mortality rate of mosquito, of these bites becomes new infective in the human population) (Esteva and Vargas, 1998). The parameters $\beta_{\mathrm{ac}}, \beta_{\mathrm{bc}}, \beta_{\mathrm{aa}}, \beta_{\mathrm{ba}}, \beta_{\mathrm{ac}}, \lambda_{\mathrm{va} 1}, \lambda_{\mathrm{va} 2}, \lambda_{\mathrm{vb} 1}$ and $\lambda_{\mathrm{vb} 2}$ are effects to the basic reproductive number of this disease as we see in (6f)-(6h). If the basic reproductive number is less or equal than one, then the infective replaces less than one, then disease dies. On the other hand, if this number is greater than one and when the susceptible fraction get large enough to birth of new susceptible, then there are secondary infections and endemic equilibrium state is occurred. As we can see in this study, the seasonal parameters such as $\varepsilon, \rho_{\mathrm{va}}$ and $\rho_{\mathrm{vb}}$ which are the measure of influence on the transmission process reflect the environment.

\section{Acknowledgment}

This study is supported by King Mongkut's Institute of Technology Ladkrabang and National Research Council of Thailand.

\section{Author's Contributions}

The work preseted here was carried out in collaboration between all authors. R. Sungchasit, P. Pongsumpun and I.M. Tang. Designed and analyzed the model. All authors have contributed to, seen and approved the manuscript.

\section{Ethics}

The authors confirm that this work is original and contains has not published elsewhere. 


\section{References}

WHO, 2009. Fact sheets: Dengue and dengue haemorrhagic.

Pongsumpun, P.P. and I. Tang, 2001. A realistic age structured transmission model for dengue hemorrhagic fever in Thailand. Math. Comput. Modell., 32: 336-340.

Syafruddin, S. and M.S.M. Noorani, 2012. SEIR Model for transmission of dengue fever in selangor Malaysia. Int. J. Mod. Phys. Conf. Ser., 9: 380-389. DOI: $10.1142 / \mathrm{S} 2010194512005454$

Kerpaninch, A., V. Watanaveeradej, R. Samakosses, S. Chumnanvanakij and T. Chulyamitporn et al., 2001. Perinatal dengue infection. Southeast Asian J. Trop Med. Publ. Health, 32: 488-493. PMID: 11944704

Kabilan, L., S. Balasubramanian, A.M. Keshava, V. Thenmozhi and C. Sehar et al., 2003. Dengue disease spectrum among infants in the 2001 dengue epidemic on Chennai, Tamil Nadu, India. J. Clin. Microbiol., 41: 3919-3921. DOI: $10.1128 /$ JCM.41.8.3919-3921.2003

Malhotra, N., C. Chanan and S. Kumar, 2006. Dengue infection in pregnancy. Int. J. Gynecol. Obstet, 94: 131-132.

Wiwanitkit, V., 2006. Dengue haemorrhagic fever in pregnancy: Appraisal on Thai cases. J. Vector Borne Disease, 43: 203-205.

Pongsumpum, P., 2011. Seasonal transmission model of dengue virus infection in Thailand. J. Basic Applied Sci. Res., 1: 1372-1379.

Joshi, V., D.T. Mourya and R.C. Sharma, 2002. Persistence of dengue -3 virus though transovarial passage in successive generations of Aedes aegypti mosquitoes. Am J. Trop Med. Hyg., 67: 158-61.

Koenraadt, C.J.M., J. Aldstadt, U. Kijchalao, A. Kengluecha and J.W. Jones et al., 2007. Spatial and temporal patterns in the recovery of Aedes aegypti (Diptera: Culicidae) populations after insecticide treatment. J. Med. Entomol., 44: 65-71. DOI: 10.1603/0022-2585(2007)44[65:SATPIT]2.0.CO;2
Hotta, S., 1998. Dengue vector mosquitoes in Japan: The role of Aedes albopictus and Aedes aegypti in the 1942-1944 dengue epidemics of Japanese Main Islands [in Japanses with English summary]. Med. Entomol. Zoollogy, 49: 267-274.

Smith, D.L., K.E. Battle, S.I. Hay, C.M. Barker and T.W. Scott et al., 2012. Macdonald and a theory for the dynamics and control mosquito-transmitted pathogens. PLos Pathog, 8: e1002588-e1002588.

Diekmann, D. and J. Heesterbeek, 2000. Mathematical Epidemiology of Infectious Disease: Model Building, Analysis and Interpretation. 1st Edn., John Wiley and Sons, Chichester, ISBN-10: 0471492418, pp: 303.

Nuraini, N., E. Soewono and K. Sidarto, 2007. Mathematical model of dengue disease transmission with severe dhf compartment. Bullentin Malaysian Math. Sci., 30: 143-157.

Yaacob, Y., 2007. Analysis of a dengue disease transmission model with immunity. Matematika, 23: 75-81.

Esteva, L. and C. Vargas, 1998. Analysis of a dengue disease transmission model. Math. Biosciences, 15: 131-151. DOI: 10.1016/S0025-5564(98)10003-2

Edelstein-Keshet, L., 1988. Mathematical Models in Biology. 1st Edn., SIAM, Norwood Mass, ISBN-10: 0898719143, pp: 586.

Chong, N.S., J.M. Tchuenche and R.J. Smith, 2013. A mathematical model of avian influenza with halfsaturated incidence. J. Theory Biosciences, 133: 23-38. DOI: 10.1007/s12064-013-0183-6 